\title{
TOPOLOGY OF MATCHING, CHESSBOARD, AND GENERAL BOUNDED DEGREE GRAPH COMPLEXES
}

\author{
MICHELLE L. WACHS*
}

In memory of Gian-Carlo Rota

\begin{abstract}
We survey results and techniques in the topological study of simplicial complexes of (di-, multi-, hyper-)graphs whose node degrees are bounded from above. These complexes have arisen is a variety of contexts in the literature. The most wellknown examples are the matching complex and the chessboard complex. The topics covered here include computation of Betti numbers, representations of the symmetric group on rational homology, torsion in integral homology, homotopy properties, and connections with other fields.
\end{abstract}

\section{Contents}

1. Introduction

2. History and connections

3. Representation of the symmetric group on homology 9

4. Hopf trace formula and Littlewood's identity 14

5. Connectivity 15

6. Torsion 16

7. General degree bounds 24

8. Digraphs, multigraphs and $B_{n}$-analogues 30

9. Hypergraphs 37

References $\quad 41$

\section{INTRODUCTION}

To every finite collection of (di-, multi-, hyper-)graphs that is closed under removal of edges, one can associate an abstract simplicial complex whose faces are the edge sets of the graphs in the collection. Graph complexes have provided an important link between combinatorics and algebra, topology and geometry; see eg., [29, 11, 47, 54, 2, 48, 37]. Here

Date: August 24, 2001, revised May 12, 2002.

* Supported in part by NSF grant DMS 0073760. 
we consider the simplicial complexes associated with the collection of subgraphs of a (di-, multi-, hyper-)graph $G$ whose node degrees are bounded from above. Some special cases which have arisen in various contexts in the recent literature are the matching complex ( $G$ is the complete graph and degree bounds are 1) and the chessboard complex ( $G$ is the complete bipartite graph and the degree bounds are 1).

The matching complex $M_{n}$ is defined to be the simplicial complex of graphs on node set $[n]:=\{1,2, \ldots, n\}$ such that each node is contained in at most one edge. A piece of $M_{7}$ (taken from [11]) is given in Figure 1.1 below. Here and throughout the paper, the vertex of $M_{n}$ labeled $i j$ represents the edge $\{i, j\}$ of the complete graph. The chessboard complex $M_{m, n}$ is defined to be the simplicial complex of bipartite graphs on partitioned node set $[m] \uplus[n]^{\prime}$, where $[n]^{\prime}=\left\{1^{\prime}, 2^{\prime}, \ldots, n^{\prime}\right\}$, such that each node is contained in at most one edge. Each $(p-1)$-face of the chessboard complex corresponds to a placement of $p$ nontaking rooks on an $m \times n$ chessboard. Indeed, a rook in the $i$ th row and $j$ th column corresponds to the edge $\left\{i, j^{\prime}\right\}$ in the bipartite graph, which corresponds to the vertex $(i, j)$ in $M_{m, n}$. It is for this reason that the name "chessboard complex" is used.

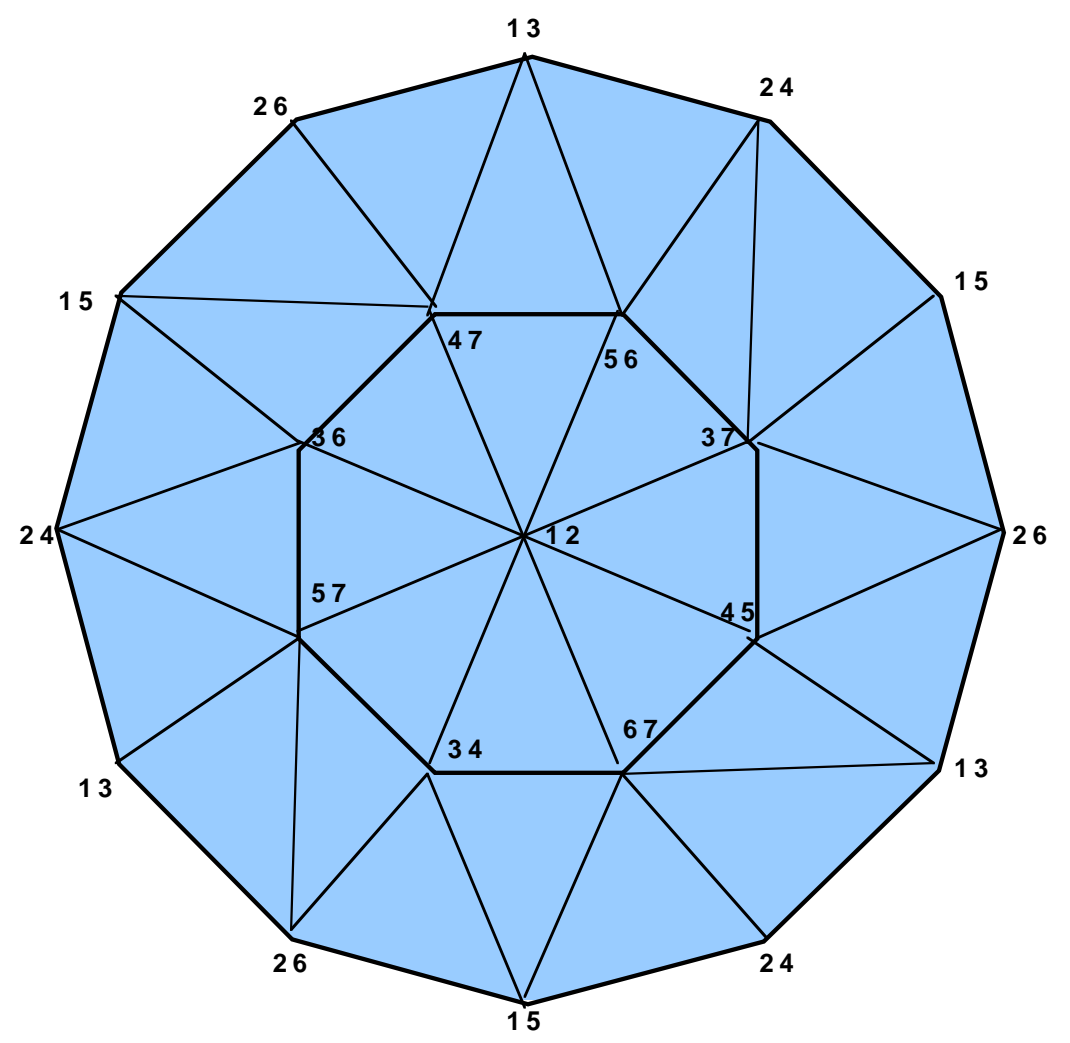

Figure 1.1: Piece of matching complex $M_{7}$ 
The symmetric group $\mathfrak{S}_{n}$ acts naturally on the matching complex $M_{n}$ by relabeling the graph nodes. For example, the transposition $(3,5)$ sends the face $\{12,34,56\}$ to $\{12,45,36\}$. This induces a representation of $\mathfrak{S}_{n}$ on the reduced simplicial homology $\tilde{H}_{p}\left(M_{n} ; k\right)$, where throughout this paper $k$ is a field of characteristic 0 . The direct product $\mathfrak{S}_{m} \times \mathfrak{S}_{n}$ acts similarly on the chessboard complex $M_{m, n}$ by relabeling the graph nodes in $[m]$ and $[n]^{\prime}$, and this induces a representation of $\mathfrak{S}_{m} \times \mathfrak{S}_{n}$ on $\tilde{H}_{p}\left(M_{m, n} ; k\right)$.

In this paper we survey results and techniques in the topological study of bounded degree (di-, multi-, hyper-)graph complexes. We discuss the earliest results in this area, dating back to the '70's, as well as the most recent developments. Many of these results are quite elegant and their proofs make use of powerful combinatorial techniques for computing homotopy type, homology and representations of the symmetric group on homology. Some of these techniques are brand new and some are classical. Because of the many contexts in which the bounded degree graph complexes have arisen in the literature, a few of the results have been discovered more than once. We attempt to sort out what's been done and to discuss the various mathematical contexts in which the graph complexes have arisen. Most of the sections end with a discussion of open problems.

We now give a more detailed description of the content of this paper. In Section 2, we discuss the contexts in which the bounded degree graph complexes have appeared in the literature of fields such as group theory, computational geometry, commutative algebra and combinatorics. The next three sections deal only with the matching and chessboard complexes and the last three sections deal with generalizations. Nearly all the results and conjectures for the matching complex (and its generalizations) have chessboard complex analogues and vice versa.

Section 3 contains a beautiful result of Bouc [11] which gives the decomposition of the representation of the symmetric group on the homology of the matching complex into irreducibles. There is an analogous result of Friedman and Hanlon [24] for the chessboard complex. These results are of fundamental importance throughout this paper. Not only do they provide combinatorial formulas for the Betti numbers of the complexes, they are even involved in the computation of torsion in integral homology. The Friedman-Hanlon proof involves an elegant application of discrete Hodge theory. It is shown by Dong and Wachs [21] that an even simpler demonstration of discrete Hodge theory can be given for the matching complex. We describe the discrete Hodge theory technique in Section 3 and sketch the Dong-Wachs and Friedman-Hanlon proofs which also involve symmetric function theory. 
In Section 4, it is observed that the Hopf trace formula for the matching complex provides a representation theoretic interpretation of a classical symmetric function identity of Littlewood. A chessboard complex analogue of the Littlewood identity is derived from the Hopf trace formula for the chessboard complex.

In Section 5 we present results of Bouc [11] and of Björner, Lovász, Vrećica and Živaljević [6] giving bounds on connectivity for the matching and chessboard complex. It was conjectured by Björner, Lovász, Vrećica and Živaljević and proved by combined work of Bouc [11] and Shareshian and Wachs [40] that these bounds are sharp. The proof is discussed in Section 6. Shellability results, conjectured by Björner, Lovász, Vrećica and Živaljević and proved by Ziegler [53] and by Shareshian and Wachs [40], are also included in Section 5.

Section 6 contains results and conjectures on torsion in the bottom nonvanishing integral homology of the matching and chessboard complexes. The first torsion results for the matching complex were obtained by Bouc [11] and were recently improved by Shareshian and Wachs [40]. Analogous results for the chessboard complex were also obtained by Shareshian and Wachs. One interesting aspect of the proof is the use of the Robinson-Schensted algorithm in constructing a basis for the top homology of the chessboard complex.

In Section 7, we present Reiner and Roberts' [37] generalizations of the decomposition results of Section 3 to general bounded degree graph complexes. Rather than describe the original proofs, we describe simpler and more elementary proofs due to Karaguezian, Reiner and Wachs [31] which use $G L_{n}(k)$ representation theory to lift the decompositions of Bouc and of Friedman and Hanlon to general bounded degree graph complexes. This lifting technique is also applied to bounded degree digraph and multigraph complexes in Section 8 and to bounded degree hypergraph complexes in Section 9. Results of Dong [19] on homotopy, predicted by the homology results of Reiner and Roberts, are also described in Section 7.

Digraph and multigraph versions of the results in the previous sections, due to Wachs [51], are presented in Section 8. The digraph and multigraph versions of the matching and chessboard complexes are viewed as "inflations" of the matching and chessboard complexes, respectively. The main tool here is a general result of Björner, Wachs and Welker [7] which expresses the homotopy type of the inflation of a simplicial complex in terms of the homotopy types of the links of the simplicial complex. Homology and equivariant homology versions of the inflation result are also used. The equivariant homology version also yields $B_{n}$-analogues of the decomposition results of Section 3 . 
Section 9 deals with bounded degree hypergraph complexes. Very few of the results of the previous sections have been generalized to hypergraph complexes. We discuss results of Björner, Lovász, Vrećica and Živaljević [6] on connectivity, results of Ksontini on connectivity and on the top nonvanishing homology, and results of Shareshian and Wachs [41] on the top nonvanishing homology.

\section{History AND CONNECTIONS}

In this section we give an overview of some of the ways in which the matching complex, the chessboard complex and general bounded degree graph complexes have arisen in the literature of various fields of mathematics.

The matching complex first appeared in a 1992 paper of Bouc [11] in connection with Brown complexes and Quillen complexes. Let $\mathcal{S}_{p}(G)$ be the poset of nontrivial $p$-subgroups of a finite group $G$ ordered by inclusion. Brown [14], [15] and Quillen [36] were interested in topological properties of the simplicial complex $\Delta\left(\mathcal{S}_{p}(G)\right)$, where $\Delta(P)$ denotes the order complex of a poset $P$, i.e., the simplicial complex of chains of $P$. Quillen showed that $\Delta\left(\mathcal{S}_{p}(G)\right)$ is contractible if $G$ has a nontrivial normal $p$-subgroup, and conjectured the converse. He reduced the problem to that of studying the elementary abelian $p$-subgroups by showing that the inclusion map from the subposet $\mathcal{A}_{p}(G)$ of nontrivial elementary abelian $p$-subgroups of $G$ to $\mathcal{S}_{p}(G)$ induces a homotopy equivalence between $\Delta\left(\mathcal{A}_{p}(G)\right)$ and $\Delta\left(\mathcal{S}_{p}(G)\right)$. The complexes $\Delta\left(\mathcal{S}_{p}(G)\right)$ and $\Delta\left(\mathcal{A}_{p}(G)\right)$ are known as the Brown complex and the Quillen complex, respectively.

Even for $G=\mathfrak{S}_{n}$ and $p=2$, not much is known about the homology of $\Delta\left(\mathcal{A}_{p}(G)\right)$ and $\Delta\left(\mathcal{S}_{p}(G)\right)$. Bouc considered the subposet $T_{n}$ of $\mathcal{S}_{2}\left(\mathfrak{S}_{n}\right)$ consisting of the 2 -subgroups of $\mathfrak{S}_{n}$ that contain a transposition, and the subposet $A_{n}$ of $\mathcal{A}_{2}\left(\mathfrak{S}_{n}\right)$ consisting of nontrivial abelian subgroups generated by transpositions. He observed that the inclusion map from $A_{n}$ to $T_{n}$ induces a homotopy equivalence between the order complexes. Since $A_{n}$ can be described combinatorially as the poset of nonempty faces of the matching complex $M_{n}$, it follows that $\Delta\left(T_{n}\right)$ and $\Delta\left(A_{n}\right)$ have the same homotopy type as $M_{n}$. The symmetric group $\mathfrak{S}_{n}$ acts on elements of $T_{n}$ and $A_{n}$ by conjugation. The homotopy equivalences between $M_{n}, \Delta\left(T_{n}\right)$ and $\Delta\left(A_{n}\right)$ are equivariant under this action.

Bouc obtained a number of interesting results on the homology of the matching complex which are discussed in Sections 3 and 6. One particularly beautiful result is a decomposition of the representation of $\mathfrak{S}_{n}$ 
on the homology of $M_{n}$ into irreducibles. This yields a combinatorial formula for the Betti numbers of $M_{n}$.

The usefulness of the matching complex $M_{n}$ in understanding the topology of the Quillen complex $\Delta\left(\mathcal{S}_{2}\left(\mathfrak{S}_{n}\right)\right)$ was recently demonstrated by Ksontini. He used simple connectivity of the matching complex $M_{n}$ for $n \geq 8$, which was proved by Bouc, to establish simple connectivity of $\Delta\left(\mathcal{S}_{2}\left(\mathfrak{S}_{n}\right)\right)$ for $n \geq 8$. It was also recently shown by Ksontini [33], Shareshian [38], and Shareshian and Wachs [41] that a hypergraph version of the matching complex discussed in Section 9 is very useful in studying the Quillen complex $\Delta\left(\mathcal{S}_{p}\left(\mathfrak{S}_{n}\right)\right)$ when $p \geq 3$.

The matching complex also appeared in a 1999 paper of Babson, Björner, Linusson, Shareshian and Welker [2] on the graph complex consisting of graphs on node set $[n]$ which are not $k$-connected. A graph is said to be $k$-connected if removal of any $j$ nodes, where $j=$ $0,1, \ldots, k-1$, results in a connected graph. When $k=1,2$, the complex arises in connection with Vassiliev knot invariants [47, 48]. It was observed in [2] that when $k=n-3$, the not $k$-connected graph complex is the Alexander dual of the matching complex $M_{n}$.

Homology of restricted block size partition posets is a subject of considerable interest in the combinatorics literature (see eg. [18, 49, 9, $8,46,44,50,12,13,45])$ and the matching complex and hypergraph matching complex provide interesting examples of such posets. Indeed, the poset of faces of $M_{n}$ is isomorphic to the poset $\Pi_{n}^{2}$ of partitions of $[n]$ in which each block has size at most 2, cf. [45]. Hence the barycentric subdivision of $M_{n}$ is isomorphic to the order complex $\Delta\left(\Pi_{n}^{2} \backslash\{\hat{0}\}\right)$ and the representation of $\mathfrak{S}_{n}$ on the homology of $M_{n}$ is isomorphic to the representation of $\mathfrak{S}_{n}$ on the homology of $\Delta\left(\Pi_{n}^{2} \backslash\{\hat{0}\}\right)$.

Prior to Bouc's study of the matching complex, the chessboard complex was first introduced in the 1979 thesis of Garst [26] dealing with Tits coset complexes. Given a group $G$ and a family of subgroups $G_{1}, \ldots, G_{m}$, the Tits coset complex $\Delta\left(G ; G_{1}, \ldots, G_{m}\right)$ is defined to be the simplicial complex whose vertices are the cosets of the subgroups and whose facets have the form $\left\{g G_{1}, \ldots, g G_{m}\right\}$, where $g \in G$. The group $G$ acts on $\Delta\left(G ; G_{1}, \ldots, G_{m}\right)$ by left multiplication. Coxeter complexes and Tits buildings are well-known examples of Tits coset complexes. By letting $G=\mathfrak{S}_{n}$ and $G_{i}=\left\{\sigma \in \mathfrak{S}_{n} \mid \sigma(i)=i\right\}$, $i=1, \ldots, m \leq n$, Garst obtained the chessboard complex

$$
M_{m, n}=\Delta\left(G ; G_{1}, \ldots, G_{m}\right),
$$

with $\mathfrak{S}_{n}$ acting on $M_{m, n}$. Garst also introduced "colored chessboard complexes" as examples of Tits coset complexes, cf., Section 8. A 
closely related class of Coxeter-like complexes, which includes the chessboard complex and the colored chessboard complex, was recently introduced and studied by Babson and Reiner [3].

Garst showed that $M_{m, n}$ is Cohen-Macaulay if and only if $2 m-1 \leq n$. Ziegler [53] strengthened this result by showing that $M_{m, n}$ is shellable if $2 m-1 \leq n$. Consequently, $M_{m, n}$ has the homotopy type of a wedge of $(m-1)$-spheres when $2 m-1 \leq n$. The number of spheres can be determined from Garst's computation of the representation of $\mathfrak{S}_{n}$ on the top homology of $M_{m, n}$ for all $m \leq n$. This computation is a precursor of Friedman and Hanlon's [24] decomposition of the representation of $\mathfrak{S}_{m} \times \mathfrak{S}_{n}$ on each homology of $M_{m, n}$ into irreducibles (Theroem 3.2).

The chessboard complex also appeared in a 1992 paper of Živaljević and Vrécica [54] in connection with some problems in computational geometry. One of these problems can be described as follows. Let $S \subseteq \mathbb{R}^{d}$ be an $n$-element set with all points in general position. An affine hyperplane $H$ is called a halving hyperplane of $S$ if $H$ is the affine hull of a $d$ element subset of $S$ and $H$ divides $S$ into subsets of equal cardinality. Let $h(S)$ be the number of halving hyperplanes of $S$ and let $h_{d}(n):=\max \left\{h(S)\left|S \subseteq \mathbb{R}^{d},\right| S \mid=n, \mathrm{~S}\right.$ in general position $\}$. The problem is to find bounds on $h_{d}(n)$. In the approach of Živaljević and Vrécica, a key role is played by connectivity properties of certain configuration spaces which are naturally associated with the so called "colored Tverberg problem". Such a configuration space can be described as the $r$-fold deleted join of the simplicial complex of partial functions in $[s] \times[t]$; i.e., the simplicial complex of all sequences $\left(f_{1}, \ldots, f_{r}\right)$ of partial functions in $[s] \times[t]$ such that if $f_{i}(a)=f_{j}(a)$ for some $a \in[s]$ then $i=j$. This simplicial complex is observed to be the $s$-fold join of the $r \times t$ chessboard complex. Since the connectivity of a join can be determined from the connectivity of its components, the connectivity of the chessboard complex determines the connectivity of the configuration space.

Motivated by the configuration space problem, Björner, Lovász, Vrécica and Živaljević [6] gave a bound on connectivity of the chessboard complex (and matching complex) and conjectured that their bound is sharp. This conjecture was settled by Shareshian and Wachs [40]. Details of these connectivity results are given in Section 5 and hypergraph versions are given in Section 9.

The study of general bounded degree graph complexes of complete graphs and complete bipartite graphs was initiated in a 2000 paper of Reiner and Roberts [37]. These complexes are interpreted as simplicial 
complexes derived from certain modules over Veronese and Segre algebras, respectively. This connection with Veronese and Segre algebras enabled Reiner and Roberts to use techniques from commutative algebra to compute the homology of the bounded degree graph complexes, generalizing the decompositions of Bouc and of Friedman and Hanlon. Details of these generalizations are presented in Section 7.

We describe the connection with the Veronese algebras given in [37] here. The connection with Segre algebras is analogous. In this discussion $k$ can be a field of arbitrary characteristic. Let $S$ be a subalgebra of a polynomial algebra $k\left[x_{1}, \ldots, x_{n}\right]$ generated by monomials $m_{1}, \ldots, m_{N}$, where this generating set is assumed to be minimal. Let $M$ be a submodule of the $S$-module $k\left[x_{1}, \ldots, x_{n}\right]$ generated by a finite set of monomials. Given $\gamma \in \mathbb{N}^{n}$ such that $x^{\gamma}:=x_{1}^{\gamma_{1}} \ldots x_{n}^{\gamma_{n}} \in M$, define a simplicial complex on vertex set $[N]$ by

$$
K_{\gamma}(M):=\left\{F \subseteq[N] \mid \frac{x^{\gamma}}{\prod_{i \in F} m_{i}} \in M\right\}
$$

This construction also appears in [16] where the simplicial complex $K_{\gamma}(M)$ is called a squarefree divisor complex.

The squarefree divisor complex is interesting because of its connection with minimal free resolutions. The surjection from $A:=k\left[z_{1}, \ldots, z_{N}\right]$ onto $S$ given by $z_{i} \mapsto m_{i}$, allows one to view $M$ as a finitely generated $A$-module. By assigning the (multi-)degree of the monomial $m_{i}$ to $z_{i}$, $A$ becomes an $\mathbb{N}^{n}$-graded algebra and $M$ an $\mathbb{N}^{n}$-graded $A$-module. This $\mathbb{N}^{n}$-grading is also carried by $\operatorname{Tor}_{i}^{A}(M, k)$. Let $\operatorname{Tor}_{i}^{A}(M, k)_{\gamma}$ denote the $\gamma^{t h}$-graded piece of $\operatorname{Tor}_{i}^{A}(M, k)$. The key result here is that

$$
\tilde{H}_{i-1}\left(K_{\gamma}(M) ; k\right) \cong \operatorname{Tor}_{i}^{A}(M, k)_{\gamma} .
$$

The Veronese subalgebra $\operatorname{Ver}(n, r, 0)$ is defined to be the subalgebra of $k\left[x_{1}, \ldots, x_{n}\right]$ generated by all monomials of degree $r$. Reiner and Roberts [37] define $\operatorname{Ver}(n, r, j)$, for $j=0, \ldots, r-1$, to be the submodule of the $\operatorname{Ver}(n, r, 0)$-module $k\left[x_{1}, \ldots, x_{n}\right]$ generated by monomials of degree $j$. It is observed in [37] that for $r=2, \gamma=1^{n}$ and $j \equiv n \bmod 2$, the squarefree divisor complex $K_{\gamma}(\operatorname{Ver}(n, r, j))$ is isomorphic to the matching complex $M_{n}$. For general $\gamma \in \mathbb{N}^{n}, r=2$ and $j \equiv|\gamma| \bmod 2$, where $|\gamma|$ is the sum of the entries of $\gamma$, the squarefree divisor complex $K_{\gamma}(\operatorname{Ver}(n, r, j))$ is isomorphic to the bounded degree graph complex $\Delta_{\gamma}$ defined in Section 7. For general $\gamma \in \mathbb{N}^{n}$, general $r$ and $j \equiv|\gamma| \bmod r$, we have $K_{\gamma}(\operatorname{Ver}(n, r, j))$ is isomorphic to the bounded degree hypergraph complex $\Delta_{r, \gamma}$ defined in Section 9.

The bounded degree graph complexes are also related to the free two-step nilpotent complex Lie algebra whose homology was computed 
first by Józefiak and Weyman [28] in 1988 and later by Sigg [42] in 1996. The homology of the rank $n$ free two-step nilpotent complex Lie algebra $L_{n}$ is a $G L_{n}(\mathbb{C})$-module whose $\gamma$-weight space is isomorphic to the homology of the bounded degree graph complex $\Delta_{\gamma}$, for all $\gamma \in \mathbb{N}^{n}$. The relationship with bounded degree graph complexes is discussed in Remark 7.3.

\section{Representation of THE SYMMETRIC GROUP ON HOMOlOGY}

Given a partition $\lambda \vdash n$, i.e., a weakly decreasing sequence of positive integers (parts) whose sum is $n$, we say $|\lambda|=n$. The length of $\lambda$, denoted $\ell(\lambda)$, is the number of parts of $\lambda$. Let $\lambda^{\prime}$ denote the conjugate of the partition $\lambda$ and let $d(\lambda)$ denote the rank of $\lambda$; i.e., the size of the main diagonal (or Durfee square) of the Ferrers diagram for $\lambda$. Frobenius notation for a partition $\lambda$, such that $d(\lambda)=d$, is the array $\left(\alpha_{1}, \ldots, \alpha_{d} \mid \beta_{1}, \ldots, \beta_{d}\right)$ where $\alpha_{i}=\lambda_{i}-i$ and $\beta_{i}=\lambda_{i}^{\prime}-i$. The Specht module (or irreducible representation) of $\mathfrak{S}_{n}$ (over $k$ ) indexed by the partition $\lambda \vdash n$, is denoted by $S^{\lambda}$.

Theorem 3.1 (Bouc [11]). For all $p, n \in \mathbb{Z}$, where $n \geq 1$, the following isomorphism of $\mathfrak{S}_{n}$-modules holds

$$
\tilde{H}_{p-1}\left(M_{n} ; k\right) \cong \mathfrak{S}_{n} \bigoplus_{\substack{\lambda: \lambda \vdash n \\ \lambda \\ \lambda=\lambda^{\prime} \\ d(\lambda)=|\lambda|-2 p}} S^{\lambda} .
$$

Theorem 3.1 was rediscovered several times, cf. [30], [37]. A result equivalent to Theorem 3.1 was stated in an earlier paper of Józefiak and Weyman [28] as a consequence of work in [27], and proved in a later paper of Sigg [42]. These results are discussed Section 7.

There is an analogous result for the chessboard complex.

Theorem 3.2 (Friedman and Hanlon [24]). For all $p, m, n \in \mathbb{Z}$, where $m, n \geq 1$, the following isomorphism of $\left(\mathfrak{S}_{m} \times \mathfrak{S}_{n}\right)$-modules holds

$$
\tilde{H}_{p-1}\left(M_{m, n} ; k\right) \cong \mathfrak{S}_{m} \times \mathfrak{S}_{n} \bigoplus_{(\lambda, \mu) \in \mathcal{R}(m, n, p)} S^{\lambda^{\prime}} \otimes S^{\mu},
$$

where $\mathcal{R}(m, n, p)$ is the set of all pairs of partitions $(\lambda \vdash m, \mu \vdash n)$ that can be obtained in the following way. Take a partition $\nu \vdash p$ that contains an $(m-p) \times(n-p)$ rectangle but contains no $(m-p+1) \times$ $\times(n-p+1)$ rectangle. Add a column of size $m-p$ to $\nu$ to obtain $\lambda$ and add a row of size $n-p$ to $\nu$ to obtain $\mu$. See Figure 3.1. 

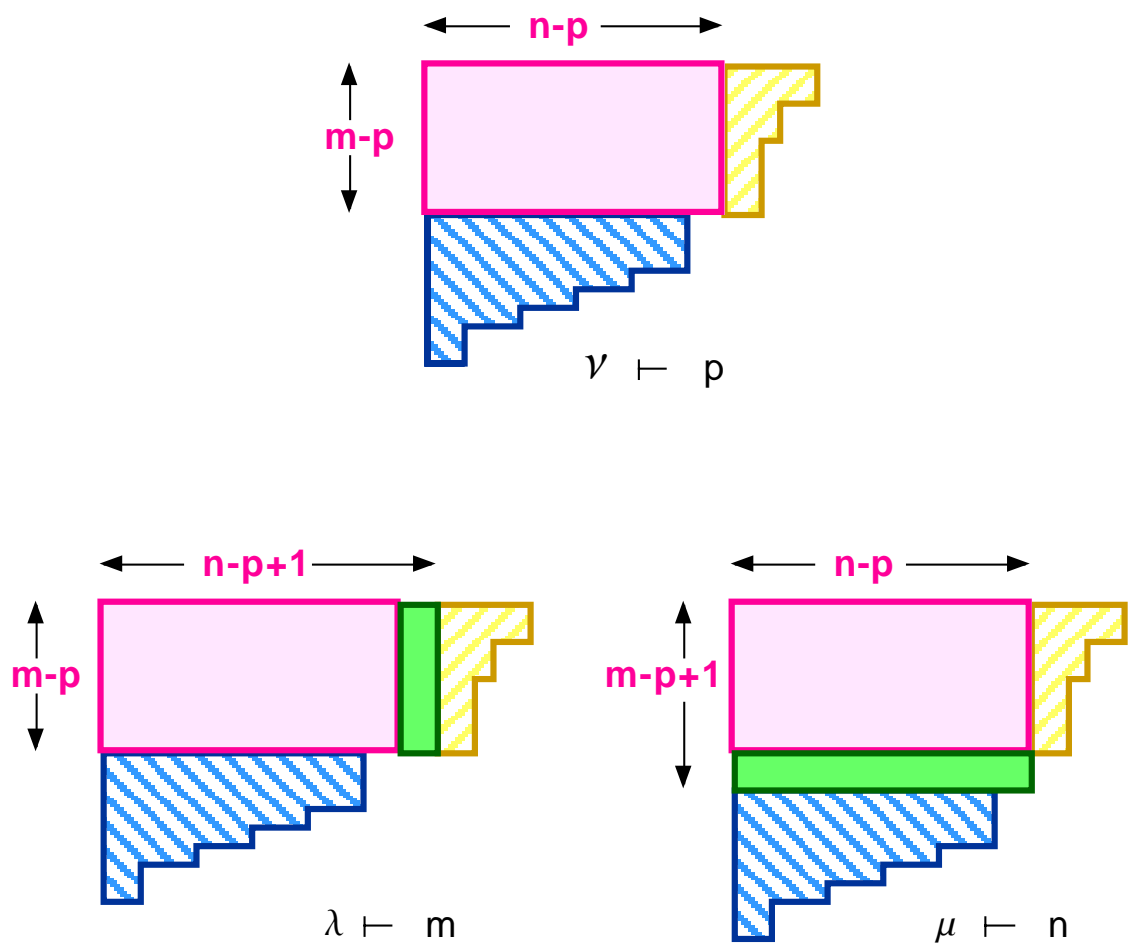

Figure 3.1

Corollary 3.3 (Garst[26]). For all $m \leq n$, the following isomorphism of $\mathfrak{S}_{n}$-modules holds

$$
\tilde{H}_{m-1}\left(M_{m, n} ; k\right) \cong \mathfrak{S}_{\substack{\lambda \vdash m \\ \lambda_{1}}} \bigoplus_{\substack{\lambda \vdash m \\ \lambda}} S^{\lambda^{*}},
$$

where $f_{\lambda}$ is the number of standard Young tableaux of shape $\lambda$ and $\lambda^{*}$ is the partition obtained from $\lambda$ by adding a part of size $n-m$.

Remark 3.4. Recall that throughout this paper the field $k$ is assumed to have characteristic 0. Bouc actually proved Theorem 3.1 for fields of finite characteristic greater than $n$ as well as for fields of characteristic 0. One can derive Theorem 3.1 for fields of characteristic greater than $n$ from the result for fields of characteristic 0 by using a long exact sequence of Bouc [11] (see the remark at the end of Section 3 of [21]). An analogous long exact sequence in [40] can be used to extend Theorem 3.2 to fields of characteristic greater than $m$ and $n$.

Friedman and Hanlon's method of proof involves a beautiful use of discrete Hodge theory. Dong and Wachs [21] showed that this method can be applied to the matching complex resulting in an elegant new 
proof of Theorem 3.1. A closely related use of this technique appears in [42]. We sketch these proofs next.

We begin with the proof of Dong and Wachs. Let $C_{p}(\Delta ; k)$ denote the augmented $p$-chain space of a finite simplicial complex $\Delta$ with coefficients in $k$. The combinatorial Laplacian $\Lambda_{p}: C_{p}(\Delta ; k) \rightarrow C_{p}(\Delta ; k)$ is defined by

$$
\Lambda_{p}=\delta_{p-1} \partial_{p}+\partial_{p+1} \delta_{p}
$$

where $\partial$ is the boundary map and $\delta$ is the coboundary map. Let $G$ be a group acting simplicially on $\Delta$. This induces a representation of $G$ on $\tilde{H}_{p}(\Delta ; k)$. The discrete analogue of Hodge theory first formulated by Kostant [32] implies that

$$
\tilde{H}_{p}(\Delta ; k) \cong_{G} \operatorname{ker} \Lambda_{p}
$$

The key observation of [21] is that when one applies the Laplacian $\Lambda_{p}$ to an oriented simplex $\gamma \in C_{p}\left(M_{n} ; k\right)$ one gets

$$
\Lambda_{p}(\gamma)=T_{n} \cdot \gamma
$$

where $T_{n}=\sum_{1 \leq i<j \leq n}(i, j) \in k \mathfrak{S}_{n}$ and $(i, j)$ denotes a transposition. By Schur's lemma $T_{n}$ acts on an irreducible representation $S^{\lambda}$ as multiplication by a scalar $c_{\lambda}$. Using a result in [35, Example 7] one sees $[24$, Lemma 1] that the scalar is simply

$$
c_{\lambda}=\sum_{i=1}^{d}\left(\left(\begin{array}{c}
\alpha_{i}+1 \\
2
\end{array}\right)-\left(\begin{array}{c}
\beta_{i}+1 \\
2
\end{array}\right)\right),
$$

where $\lambda=\left(\alpha_{1}, \ldots, \alpha_{d} \mid \beta_{1}, \ldots, \beta_{d}\right)$ in Frobenius notation.

To decompose $C_{p}\left(M_{n} ; k\right)$ into irreducibles, one first observes that

$$
C_{p-1}\left(M_{n} ; k\right) \cong_{\mathfrak{S}_{n}}\left(S^{1^{p}} \circ S^{2}\right) \cdot S^{n-2 p},
$$

where $\circ$ denotes the composition product (or plethysm) and . denotes the induction product. There is a symmetric function formula of Littlewood for the composition product, cf., [35, I 5 Ex. 9b],

$$
\prod_{i \leq j}\left(1-x_{i} x_{j}\right)=\sum_{\nu \in \mathcal{B}}(-1)^{|\nu| / 2} s_{\nu}
$$

where $\mathcal{B}$ is the set of all partitions of form $\left(\alpha_{1}+1, \ldots, \alpha_{d}+1 \mid \alpha_{1}, \ldots, \alpha_{d}\right)$ for some $d$, and $s_{\nu}$ is the Schur function indexed by $\nu$. This and Pieri's rule for the induction product yield the decomposition into irreducibles:

$$
C_{p-1}\left(M_{n} ; k\right)=\bigoplus_{\lambda \in A_{n}} a_{\lambda}^{p} S^{\lambda}
$$

where

$$
A_{n}=\left\{\left(\alpha_{1}, \ldots, \alpha_{d} \mid \beta_{1}, \ldots, \beta_{d}\right) \vdash n \mid d \geq 1, \alpha_{i} \geq \beta_{i} \forall i \in[d]\right\}
$$


and $a_{\lambda}^{p}$ is a nonnegative integer. If $\lambda$ is self-conjugate then

$$
a_{\lambda}^{p}= \begin{cases}1 & \text { if } d(\lambda)=n-2 p \\ 0 & \text { otherwise }\end{cases}
$$

Clearly for $\lambda \in A_{n}, c_{\lambda}=0$ if and only if $\lambda$ is self-conjugate. It follows that

$$
\operatorname{ker} \Lambda_{p-1} \cong \bigoplus_{\substack{\lambda: \lambda \vdash n \\ \lambda=\lambda^{\prime}}} a_{\lambda}^{p} S^{\lambda} \cong \bigoplus_{\substack{\lambda: \lambda \vdash n \\ \lambda=\lambda^{\prime} \\ d(\lambda)=n-2 p}}^{\bigoplus} S^{\lambda} .
$$

Theorem 3.1 now follows by discrete Hodge theory. This method also yields:

Theorem 3.5 (Dong and Wachs [21]). All the eigenvalues of the Laplacian on the matching complex are nonnegative integers. Moreover for each eigenvalue $c$, the c-eigenspace of the Laplacian $\Lambda_{p-1}$ on $M_{n}$ decomposes into the following direct sum of irreducible $\mathfrak{S}_{n}$ modules

$$
\bigoplus_{\substack{\lambda \in A_{n} \\ c_{\lambda}=c}} a_{\lambda}^{p} S^{\lambda},
$$

where $a_{\lambda}^{p}$ is the number of partitions $\nu \vdash 2 p$ of the form $\nu=\left(\beta_{1}+\right.$ $\left.1, \ldots, \beta_{d}+1 \mid \beta_{1}, \ldots, \beta_{d}\right)$ such that $\lambda / \nu$ is a horizontal strip.

The following is a restatement of Theorem 4 and Corollary 2 of Friedman and Hanlon [24]. It contains a simplification of an expression appearing in Theorem 4 .

Theorem 3.6 (Friedman and Hanlon [24]). All the eigenvalues of the Laplacian on the chessboard complex are nonnegative integers. Moreover for each eigenvalue $c$, the c-eigenspace of the Laplacian $\Lambda_{p-1}$ on $M_{m, n}$ decomposes into the following direct sum of irreducible $\mathfrak{S}_{m} \times \mathfrak{S}_{n}$ modules:

$$
\bigoplus_{\substack{\lambda \vdash m \\
\mu \vdash n \\
c_{\lambda}+c_{\mu}=c+\left(\begin{array}{c}
n+m \\
2
\end{array}\right)-2 m n}} a_{\lambda, \mu}^{p} S^{\lambda} \otimes S^{\mu},
$$

where $a_{\lambda, \mu}^{p}$ is the number of partitions $\nu \vdash p$ such that $\lambda / \nu^{\prime}$ and $\mu / \nu$ are horizontal strips, and $c_{\lambda}$ and $c_{\mu}$ are defined in (3.1). 
Friedman and Hanlon show that Theorem 3.2 can be obtained from Theorem 3.6, by establishing the following identity for $\left(\lambda^{\prime}, \mu\right) \in R(m, n, p)$ :

$$
c_{\lambda}+c_{\mu}=\left(\begin{array}{c}
m-p \\
2
\end{array}\right)+\left(\begin{array}{c}
n-p \\
2
\end{array}\right)-p-(m-p)(n-p)
$$

Note that the expression on the right of (3.4) simplifies to $\left(\begin{array}{c}m+n \\ 2\end{array}\right)-2 m n$. We also have that if $\left(\lambda^{\prime}, \mu\right) \in R(m, n, p)$ then $a_{\lambda, \mu}^{p}=1$. Hence the irreducible $S^{\lambda} \otimes S^{\mu}$ has multiplicity 1 in the kernel of the Laplacian. The proof is completed by showing that $c_{\lambda}+c_{\mu}$ is greater than the expression on the right of (3.4) for all pairs $(\lambda, \mu) \notin R(m, n, p)$ for which $a_{\lambda, \mu}^{p} \neq 0$.

We will now sketch a slightly simplified version of Friedman and Hanlon's proof of Theorems 3.6. A straight-forward analysis of the behavior of the Laplacian on an oriented simplex $\gamma$ of the chessboard complex yields

$$
\begin{aligned}
\Lambda_{p-1}(\gamma)= & \left(T_{m} \otimes 1+1 \otimes T_{n}+p+(n-p)(m-p)\right. \\
& \left.-\left(\begin{array}{c}
n-p \\
2
\end{array}\right)-\left(\begin{array}{c}
m-p \\
2
\end{array}\right)\right) \cdot \gamma \\
= & \left(T_{m} \otimes 1+1 \otimes T_{n}-\left(\begin{array}{c}
m+n \\
2
\end{array}\right)+2 m n\right) \cdot \gamma .
\end{aligned}
$$

Since $T_{m} \otimes 1$ acts as multiplication by the scalar $c_{\lambda}$ on the irreducible $S^{\lambda} \otimes S^{\mu}$ and $1 \otimes T_{n}$ acts as multiplication by the scalar $c_{\mu}$, the decomposition of the eigenspaces can be obtained by decomposing $C_{p-1}\left(M_{m, n} ; k\right)$ into irreducibles. Observe that

$$
C_{p-1}\left(M_{m, n} ; k\right) \cong_{\mathfrak{S}_{m} \times \mathfrak{S}_{n}}\left(S^{1^{p}} \circ\left(S^{1} \otimes S^{1}\right)\right) \cdot\left(S^{m-p} \otimes S^{n-p}\right) .
$$

By the symmetric function formula $\left(4.3^{\prime}\right)$ in $[35$, I 4$]$ we have

$$
C_{p-1}\left(M_{m, n} ; k\right) \cong_{\mathfrak{S}_{m} \times \mathfrak{S}_{n}}\left(\bigoplus_{\nu \vdash p} S^{\nu^{\prime}} \otimes S^{\nu}\right) \cdot\left(S^{m-p} \otimes S^{n-p}\right) .
$$

The decomposition of $C_{p-1}\left(M_{m, n} ; k\right)$ into irreducibles now follows from Pieri's rule.

Problem 3.7. Find a natural basis for the (co)homology of $M_{n}$ indexed by standard Young tableaux of self-conjugate shape. Find an analogous basis for $\tilde{H}_{p-1}\left(M_{m, n} ; k\right)$ indexed by pairs of standard Young tableaux in $\mathcal{R}(m, n, p)$. In [40] such a basis for the top homology (and cohomology) of the chessboard complex is constructed; see Section 6.3. 


\section{Hopf TRACE FORMUlA AND LitTleWOOD's IDENTITY}

There is another symmetric function identity of Littlewood [34, p.238], namely

$$
\prod_{i \leq j}\left(1-x_{i} x_{j}\right) \prod_{i}\left(1-x_{i}\right)^{-1}=\sum_{\lambda=\lambda^{\prime}}(-1)^{\frac{|\lambda|-d(\lambda)}{2}} s_{\lambda} .
$$

A combinatorial proof of the Littlewood identities (3.3) and (4.1) involving matchings is given by Burge [17]. Macdonald [35, I 5 Ex. 9b,c] observes that Littlewood's identities (3.3) and (4.1) can be interpreted as the Weyl denominator formula for the root systems $C_{n}$ and $B_{n}$, respectively.

Using Theorem 3.1 one can also interpret Littlewood's formula (4.1) as the Hopf trace formula for the matching complex. Indeed, by extracting the degree $n$ terms one gets

$$
\sum_{p \geq 0}(-1)^{p} e_{p}\left[h_{2}\right] h_{n-2 p}=\sum_{p \geq 0}(-1)^{p} \sum_{\substack{\lambda: \lambda \vdash n \\ \lambda=\lambda^{\prime} \\ d(\lambda)=n-2 p,}} s_{\lambda}
$$

where $e_{p}$ and $h_{r}$ are the elementary and complete homogeneous symmetric functions, resp., and $f[g]$ denotes plethysm. The representation theoretic version (via the Frobenius characteristic) of (4.2) is

$$
\bigoplus_{p \geq 0}(-1)^{p}\left(S^{1^{p}} \circ S^{2}\right) \cdot S^{n-2 p} \cong \mathfrak{S}_{n} \bigoplus_{p \geq 0}(-1)^{p} \bigoplus_{\substack{\lambda: \lambda \vdash n \\ \lambda=\lambda^{\prime} \\ d(\lambda)=n-2 p .}} S^{\lambda} .
$$

By (3.2) and Theorem 3.1, this is precisely the Hopf trace formula for the matching complex:

$$
\bigoplus_{p \geq 0}(-1)^{p} C_{p-1}\left(M_{n} ; \mathbb{C}\right) \cong_{\mathfrak{S}_{n}} \bigoplus_{p \geq 0}(-1)^{p} \tilde{H}_{p-1}\left(M_{n} ; C\right)
$$

This interpretation of Littlewood's formula can be found in [43, Solutions 7.29a]. Representation theoretic interpretations of Littlewood's formula involving Lie algebra homology are given by Józefiak and Weyman [28] and by Sigg [42].

We now present the chessboard complex analogue of Littlewood's formula (4.1). By Theorem 3.2 and (3.5), the Hopf trace formula for the chessboard complex is

$$
\bigoplus_{p \geq 0}(-1)^{p}\left(S^{1^{p}} \circ\left(S^{1} \otimes S^{1}\right)\right) \cdot\left(S^{m-p} \otimes S^{n-p}\right) \cong \mathfrak{S}_{m} \times \mathfrak{S}_{n} \bigoplus_{p \geq 0}(-1)^{p} \bigoplus_{(\lambda, \mu) \in \mathcal{R}(m, n, p)} S^{\lambda^{\prime}} \otimes S^{\mu} .
$$


By taking the Frobenius characteristic and summing over all $m, n$ one gets

$\prod_{i, j}\left(1-x_{i} y_{j}\right) \prod_{i}\left(1-x_{i}\right)^{-1}\left(1-y_{i}\right)^{-1}=\sum_{(\lambda, \mu) \in \mathcal{R}}(-1)^{p(\lambda, \mu)} s_{\lambda^{\prime}}(x) s_{\mu}(y)$,

where $\mathcal{R}=\biguplus_{m, n, p} \mathcal{R}(m, n, p)$ and $p(\lambda, \mu)$ is the unique integer $p$ such that $(\lambda, \mu) \in \mathcal{R}(m, n, p)$.

\section{Connectivity}

Define the connectivity degree of a simplicial complex $\Delta$ to be the largest $j \leq \operatorname{dim} \Delta$ such that $\Delta$ is $j$-connected.

For $1 \leq m \leq n$, let

$$
\nu_{n}=\left\lfloor\frac{n+1}{3}\right\rfloor-1 \quad \text { and } \quad \nu_{m, n}=\min \left\{m,\left\lfloor\frac{m+n+1}{3}\right\rfloor\right\}-1 .
$$

Theorem 5.1 ( Björner, Lovász, Vrécica, Z̆ivaljević [6]). For $n \geq 2$, the connectivity degree of the matching complex $M_{n}$ is at least $\nu_{n}-1$. For $1 \leq m \leq n$, the connectivity degree of the chessboard complex $M_{m, n}$ is at least $\nu_{m, n}-1$.

This result, just for the matching complex, was also proved by Bouc [11]. In [6] the result is proved using a nerve lemma. It is conjectured in [6] that these connectivity bounds are sharp. Theorems 3.1 and 3.2 imply the validity of the conjecture only for certain values of $m$ and $n$ because these theorems determine the Betti numbers and not the torsion. It turns out that there is torsion in $\tilde{H}_{\nu_{n}}\left(M_{n} ; \mathbb{Z}\right)$ and $\tilde{H}_{\nu_{m, n}}\left(M_{m, n} ; \mathbb{Z}\right)$ for $n$ sufficiently large and $m$ sufficiently close to $n$. Torsion is discussed in the next section. The following theorem settles the conjecture.

Theorem 5.2 (Bouc [11], Shareshian and Wachs [40]). For $n \geq 3$,

$$
\tilde{H}_{\nu_{n}}\left(M_{n} ; \mathbb{Z}\right) \neq 0,
$$

and for $2 \leq m \leq n$,

$$
\tilde{H}_{\nu_{m, n}}\left(M_{m, n} ; \mathbb{Z}\right) \neq 0 .
$$

In [11] Bouc establishes (5.1) for the cases $n \equiv 0,1 \bmod 3$. It is observed in [40] that the $2 \bmod 3$ case follows from a long exact sequence in [11] and the 1 mod 3 case. Equation (5.2) is proved in [40] by means of an analogous long exact sequence which is used to reduce the $m+n \equiv$ $2 \bmod 3$ case to the $1 \bmod 3$ case. The $m+n \equiv 0,1 \bmod 3$ cases are 
handled by showing that certain nonzero elements of $\tilde{H}_{\nu_{m+n}}\left(M_{m+n} ; \mathbb{Z}\right)$ are in $\tilde{H}_{\nu_{m, n}}\left(M_{m, n} ; \mathbb{Z}\right)$ when $2 m-1>n$ and by applying Theorem 3.2 when $2 m-1 \leq n$.

A consequence of Theorem 5.2 is that the depth of the StanleyReisner ring of the matching complex $M_{n}$ is $\nu_{n}+1$ and of the chessboard complex $M_{m, n}$ is $\nu_{m, n}+1$ (see [6]).

The $r$-skeleton of a simplicial complex $\Delta$ is denoted by $\Delta^{(r)}$ and is defined to be the subcomplex of $\Delta$ generated by faces of dimension $r$ or less. A consequence of Theorem 5.1 is that the skeleta $M_{n}^{\left(\nu_{n}\right)}$ and $M_{m, n}^{\left(\nu_{m, n}\right)}$ are homotopy Cohen-Macaulay. This led Björner, Lovász, Vrécica and Živaljević to conjecture that these skeleta are shellable [6]. This conjecture was proved first for the chessboard complex by Ziegler [53] by establishing vertex decomposability, a condition stronger than shellability. Shareshian and Wachs [40] [39] settled the conjecture for the matching complex by exhibiting an explicit shelling order of the facets.

Theorem 5.3 (Ziegler [53], Shareshian and Wachs [40][39]). For all $n \geq$ 2 , the skeleton $M_{n}^{\left(\nu_{n}\right)}$ is shellable, and for all $1 \leq m \leq n$, the skeleton $M_{m, n}^{\left(\nu_{m, n}\right)}$ is shellable.

Ziegler [53] also establishes vertex decomposability of skeleta of chessboard complexes for certain classes of nonrectangular shapes.

Problem 5.4. Show that $M_{n}^{\left(\nu_{n}\right)}$ is vertex decomposable. ${ }^{1}$

\section{TORSION}

In this section we report on joint work with Shareshian [40][39] dealing with torsion in the bottom nonvanishing integral homology of the matching and chessboard complexes. Recall from the previous section that the bottom nonvanishing integral reduced homology of $M_{n}$ and $M_{m, n}$ occurs in degree $\nu_{n}$ and $\nu_{m, n}$, respectively. We will see that these bottom nonvanishing homology groups have a very nice structure.

As is customary, we suppress $\mathbb{Z}$ in our notation for integral homology of $\Delta$ and write $\tilde{H}_{p}(\Delta)$ instead of $\tilde{H}_{p}(\Delta ; \mathbb{Z})$.

6.1. The matching complex. It follows from Theorem 3.1 that the integral homology group $\tilde{H}_{\nu_{n}}\left(M_{n}\right)$ is finite if and only if $n=7,10$ or $n \geq 12$. This is because the only values of $n$, for which there is a selfconjugate partition $n$ whose rank is $n-2\left(\nu_{n}+1\right)$, are $n \leq 6, n=8,9,11$.

\footnotetext{
${ }^{1}$ See New Developments Section at the end of the paper.
} 
One can see 3 -torsion in $\tilde{H}_{1}\left(M_{7}\right)$ by looking at Figure 1.1. The union of the triangles shown is bounded by the 1-chain $3 \rho$ where

$$
\rho=(13,24)+(24,15)+(15,26)+(26,13) .
$$

If the cycle $\rho$ is not a boundary, then $\tilde{H}_{1}\left(M_{7}\right)$ has 3 -torsion. This is indeed the case. In fact it turns out that $\rho$ generates the group $\tilde{H}_{1}\left(M_{7}\right)$.

The integral homology groups of $M_{n}$, for $n \leq 12$, are shown in Table 6.1 which first appeared in [2]. They were computed using a computer program of Heckenbach which was later updated by Dumas, Heckenbach, Saunders and Welker [22]. The table shows that $M_{n}$ can have torsion and it suggests that there is $p$-torsion only for the prime $p=3$, see [2, Question 9.2]. Bouc [11] showed that this is indeed the case for the bottom nonvanishing homology of $M_{n}$ when $n \equiv 0,1 \bmod 3$. Shareshian and Wachs [40] extend this to all $n$.

\begin{tabular}{|c||c|c|c|c|c|c|}
\hline$n \backslash i$ & 0 & 1 & 2 & 3 & 4 & 5 \\
\hline \hline 2 & 0 & 0 & 0 & 0 & 0 & 0 \\
\hline 3 & $\mathbb{Z}^{2}$ & 0 & 0 & 0 & 0 & 0 \\
\hline 4 & $\mathbb{Z}^{2}$ & 0 & 0 & 0 & 0 & 0 \\
\hline 5 & 0 & $\mathbb{Z}^{6}$ & 0 & 0 & 0 & 0 \\
\hline 6 & 0 & $\mathbb{Z}^{16}$ & 0 & 0 & 0 & 0 \\
\hline 7 & 0 & $\mathbb{Z}_{3}$ & $\mathbb{Z}^{20}$ & 0 & 0 & 0 \\
\hline 8 & 0 & 0 & $\mathbb{Z}^{132}$ & 0 & 0 & 0 \\
\hline 9 & 0 & 0 & $\mathbb{Z}^{42} \oplus \mathbb{Z}_{3}^{8}$ & $\mathbb{Z}^{70}$ & 0 & 0 \\
\hline 10 & 0 & 0 & $\mathbb{Z}_{3}$ & $\mathbb{Z}^{1216}$ & 0 & 0 \\
\hline 11 & 0 & 0 & 0 & $\mathbb{Z}^{1188} \oplus Z_{3}^{45}$ & $\mathbb{Z}^{252}$ & 0 \\
\hline 12 & 0 & 0 & 0 & $\mathbb{Z}_{3}^{56}$ & $\mathbb{Z}^{12440}$ & 0 \\
\hline
\end{tabular}

Table 6.1: Integral homology of matching complex $\tilde{H}_{i}\left(M_{n}\right)$

Theorem 6.1 (Bouc[11]).

(i) If $n \equiv 1 \bmod 3$ and $n \geq 7$ then $\tilde{H}_{v_{n}}\left(M_{n}\right) \cong \mathbb{Z}_{3}$.

(ii) If $n \equiv 0 \bmod 3$ and $n \geq 12$ then $\tilde{H}_{v_{n}}\left(M_{n}\right)$ is a nontrivial 3-group of exponent at most 9 .

Theorem 6.2 (Shareshian and Wachs [40]). For $n \geq 12$ (except possibly $n=14$ ),

$$
\tilde{H}_{\nu_{n}}\left(M_{n}\right) \cong \mathbb{Z}_{3}^{r_{n}}
$$

where $r_{n} \geq 1$.

To prove Theorem 6.1 , Bouc first computes $\tilde{H}_{\nu_{7}}\left(M_{7}\right)$ to be $\mathbb{Z}_{3}$. He then constructs a long exact sequence of homology from which it follows 
immediately that for $n \equiv 0,1 \bmod 3$, the homology group $\tilde{H}_{\nu_{n}}\left(M_{n}\right)$ is spanned by cycles of the form

$$
\alpha * \beta
$$

where

$$
\alpha \in \tilde{H}_{0}\left(M_{\{a, b, c\}}\right) \text { and } \beta \in \tilde{H}_{\nu_{n-3}}\left(M_{[n] \backslash\{a, b, c\}}\right)
$$

for some distinct $a, b, c \in[n]$. Here, $*$ denotes an operation associated with concatenation of oriented simplexes, and $M_{A}$ denotes the simplicial complex of degree 1 graphs on node set $A$. Since $3(\alpha * \beta)=\alpha * 3 \beta$, one can conclude by induction that $\tilde{H}_{\nu_{n}}\left(M_{n}\right)$ has exponent at most 3 in the case that $n \equiv 1 \bmod 3$ and $n \geq 7$. Bouc does some additional work to conclude that the rank is 1 and still more work to handle the case $n \equiv 0 \bmod 3$.

The computer calculation $\tilde{H}_{4}\left(M_{12}\right)=\mathbb{Z}_{3}^{56}$ yields the improvement of Bouc's result for the case $n \equiv 0 \bmod 3$. It provides the base step of the induction for this case.

Although it is easy to use Bouc's long exact sequence to establish the nontriviality of $\tilde{H}_{\nu_{n}}\left(M_{n}\right)$ in the case $n \equiv 2 \bmod 3$, the fact that the exponent of $\tilde{H}_{\nu_{n}}\left(M_{n}\right)$ is 3 in the case that $n \equiv 2 \bmod 3$ and $n \geq 17$ does not follow so easily from Bouc's long exact sequence. It is shown in [40] that if $n \equiv 2 \bmod 3$ then $\tilde{H}_{\nu_{n}}\left(M_{n}\right)$ is spanned by cycles of the form

$$
\alpha * \beta
$$

where

$$
\alpha \in \tilde{H}_{1}\left(M_{\{a, b, c, d, e\}}\right) \text { and } \beta \in \tilde{H}_{\nu_{n-5}}\left(M_{[n] \backslash\{a, b, c, d, e\}}\right)
$$

for some distinct $a, b, c, d, e \in[n]$. This reduces the $2 \bmod 3$ case to the $0 \bmod 3$ case. Indeed, for $n \geq 17$ we have $3(\alpha * \beta)=\alpha * 3 \beta=0$, since $n-5 \equiv 0 \bmod 3$ and $n-5 \geq 12$. Hence the exponent of $\tilde{H}_{\nu_{n}}\left(M_{n}\right)$ is 3 for $n \equiv 2 \bmod 3$ and $n \geq 17$. Note that this argument does not work for $\tilde{H}_{\nu_{14}}\left(M_{14}\right)$ since $\tilde{H}_{\nu_{9}}\left(M_{9}\right)$ has a nontrivial free part. The homology of $M_{n}$ has been computed using the software of [22] only up to $n=13$.

6.2. The chessboard complex. Table 6.2, which was obtained by using the software of [22], gives the bottom nonvanishing homology groups of the chessboard complex $M_{m, n}$ for $2 \leq m \leq n \leq 7$. It is clear that bottom nonvanishing homology is free when $2 m-1 \leq n$. Indeed, in this case $\nu_{m, n}=m-1=\operatorname{dim} M_{m, n}$. Hence the bottom nonvanishing homology is the same as the top homology which has to be free. It follows that when $2 m-1 \leq n$, the bottom nonvanishing homology can be computed using the Friedman-Hanlon decomposition (Theorem 3.2). 


\begin{tabular}{|c||c|c|c|c|c|c|}
\hline$m \backslash n$ & 2 & 3 & 4 & 5 & 6 & 7 \\
\hline \hline 2 & $\mathbb{Z}$ & $\mathbb{Z}$ & $\mathbb{Z}^{5}$ & $\mathbb{Z}^{11}$ & $\mathbb{Z}^{19}$ & $\mathbb{Z}^{29}$ \\
\hline 3 & & $\mathbb{Z}^{4}$ & $\mathbb{Z}^{2}$ & $\mathbb{Z}^{14}$ & $\mathbb{Z}^{47}$ & $\mathbb{Z}^{104}$ \\
\hline 4 & & & $\mathbb{Z}^{15}$ & $\mathbb{Z}^{20}$ & $\mathbb{Z}^{5}$ & $\mathbb{Z}^{225}$ \\
\hline 5 & & & & $\mathbb{Z}_{3}$ & $\mathbb{Z}^{152}$ & $\mathbb{Z}^{98}$ \\
\hline 6 & & & & & $\mathbb{Z}^{25} \oplus \mathbb{Z}_{3}^{10}$ & $\mathbb{Z}_{3}$ \\
\hline 7 & & & & & & $\mathbb{Z}^{588} \oplus \mathbb{Z}_{3}^{66}$ \\
\hline
\end{tabular}

Table 6.2: Bottom nonvanishing homology $\tilde{H}_{\nu_{m, n}}\left(M_{m, n}\right)$

Note that the only finite groups appearing in Table 6.2 are

$$
\tilde{H}_{\nu_{5,5}}\left(M_{5,5}\right)=\tilde{H}_{\nu_{6,7}}\left(M_{6,7}\right)=\mathbb{Z}_{3} .
$$

This suggests analogues of Theorems 6.1 and 6.2 .

Theorem 6.3 (Shareshian and Wachs [40]). Let $m \leq n$.

(i) If $m+n \equiv 1 \bmod 3$ and $5 \leq n \leq 2 m-5$ then $\tilde{H}_{v_{m, n}}\left(M_{m, n}\right) \cong \mathbb{Z}_{3}$.

(ii) If $m+n \equiv 0 \bmod 3$ and $9 \leq n \leq 2 m-9$ then $\tilde{H}_{v_{m, n}}\left(M_{m, n}\right)$ is a nontrivial 3-group of exponent at most 9.

(iii) If $m+n \equiv 2 \bmod 3$ and $13 \leq n \leq 2 m-13$ then $\tilde{H}_{v_{m, n}}\left(M_{m, n}\right)$ is a nontrivial 3-group of exponent at most 9.

It is conjectured in [40] that the exponent of $\tilde{H}_{v_{m, n}}\left(M_{m, n}\right)$ is in fact 3 in all cases. The following theorem states that in order to establish this conjecture one needs only check it for $m=n=9$. However the computer, using the software of [22], is not yet able to produce results for these values.

Theorem 6.4 (Shareshian and Wachs [40]). If $\tilde{H}_{\nu_{9,9}}\left(M_{9,9}\right)$ has exponent 3 then so does $\tilde{H}_{v_{m, n}}\left(M_{m, n}\right)$, for all $m$ and $n$ that satisfy the conditions of Theorem 6.3

The proofs of these theorems are similar to, but considerably more difficult than the proofs of their counterparts for the matching complex (Theorems 6.1 and 6.2). It is straightforward to construct a chessboard complex analogue of Bouc's long exact sequence. However, it is not as easy to exploit this sequence as it was to use the original sequence. In fact, it is necessary to understand the top homology of the chessboard complex in order to proceed with the induction process used in the proof.

We briefly explain how top homology enters the proof for the $m+n \equiv$ $0,1 \bmod 3$ cases. For $m \leq n \leq 2 m-2$ the long exact sequence readily 
yields a decomposition of generating cycles of $\tilde{H}_{\nu_{m, n}}\left(M_{m, n}\right)$ into cycles of the form $\alpha * \beta$, where

$$
\alpha \in \tilde{H}_{0}\left(M_{I, J}\right) \text { and } \beta \in \tilde{H}_{\nu_{m-|I|, n-|J|}}\left(M_{[m]-I,[n]-J}\right),
$$

for $I \subseteq[m], J \subseteq[n]$ such that

$$
|I|=1,|J|=2 \text { or }|I|=2,|J|=1 .
$$

Again we have

$$
3(\alpha * \beta)=\alpha * 3 \beta .
$$

However, if $|I|=2$ and $|J|=1$ then $m-2$ and $n-1$ might not be close enough to satisfy the hypothesis of Theorem 6.3 which means that the induction hypothesis $3 \beta=0$ cannot be invoked. Indeed, $\tilde{H}_{\nu_{m-2, n-1}}\left(M_{[m]-|I|,[n]-|J|}\right)$ could be free top homology. To overcome this problem, it is necessary to find generating cycles for the top homology of the chessboard complex which decompose into smaller cycles. This is accomplished by the construction of a basis for the top homology of the chessboard complex.

\subsection{Basis for top (co)homology of the chessboard complex.} It follows immediately from Corollary 3.3 that the rank of the top homology of the chessboard complex $M_{m, n}$ is the number of pairs of standard Young tableaux $(S, T)$ such that $S$ has $m$ cells, $T$ has $n$ cells and the shape of $S$ is the same as the shape of $T$ minus the first row. Let $\mathcal{P}_{m, n}$ be the set of such pairs of standard tableaux. We construct for each $(S, T) \in \mathcal{P}_{m, n}$, an element $\rho(S, T) \in \tilde{H}_{m-1}\left(M_{m, n}\right)$ and an element $\gamma(S, T) \in \tilde{H}^{m-1}\left(M_{m, n}\right)$, and show that these elements form bases for homology and cohomology, respectively. This construction is based on the classical Robinson-Schensted correspondence.

Let $(S, T) \in \mathcal{P}_{m, n}$. First add a cell with entry $\infty$ to the bottom of each of the first $n-m$ columns (some may be empty) of $S$ to obtain a semistandard tableau $S^{*}$ of the same shape as $T$. (Here $\infty$ represents a number larger than $m$.) The inverse of the Robinson-Schensted bijection applied to $\left(S^{*}, T\right)$ produces a permutation $\sigma$ of the multiset $\left\{1,2, \ldots, m, \infty^{n-m}\right\}$. The multiset permutation $\sigma$ corresponds naturally to the oriented simplex (i.e., matching) of $M_{m, n}$ given by

$$
\left(\left(\sigma\left(i_{1}\right), i_{1}\right),\left(\sigma\left(i_{2}\right), i_{2}\right), \ldots,\left(\sigma\left(i_{m}\right), i_{m}\right)\right),
$$

where $\sigma\left(i_{1}\right) \sigma\left(i_{2}\right) \cdots \sigma\left(i_{m}\right)$ is the subword of $\sigma=\sigma(1) \sigma(2) \cdots \sigma(n)$ obtained by removing the $\infty$ 's. This oriented simplex is clearly a cocycle since it is in the top dimension. Let $\gamma(S, T)$ be the coset of the coboundary group $B^{m-1}\left(M_{m, n}\right):=\operatorname{Im} \delta_{m-2}$ that contains this oriented simplex. 
We demonstrate the procedure for constructing $\gamma(S, T)$ by letting $(S, T)$ be the pair of tableaux given in Figure 6.1. After applying the inverse of Robinson-Schensted to $\left(S^{*}, T\right)$ we have the multiset permutation $\infty \infty 2 \infty 4 \infty 31$. The oriented simplex that corresponds to this multiset permutation is $((2,3),(4,5),(3,7),(1,8))$. Hence, $\gamma(S, T)$ is the coset of $B^{3}\left(M_{4,8}\right)$ that contains the oriented simplex

$$
((2,3),(4,5),(3,7),(1,8)) \text {. }
$$

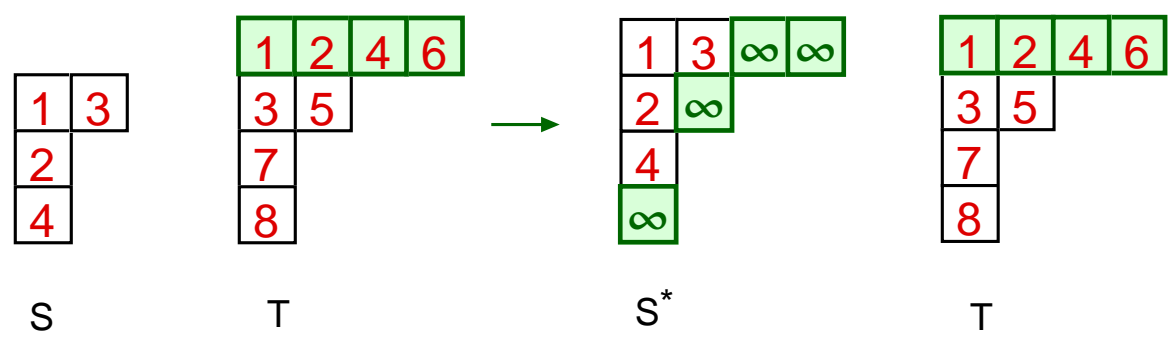

Figure 6.1

The construction of the cycles is a bit more involved. Recall that in the inverse Robinson-Schensted procedure, an entry "pops" from a cell in the top row of the left tableau when an entry is "crossed out" of the right tableau. For each top cell, we must keep track of the entries of $S^{*}$ that are popped and the corresponding entries of $T$ that are crossed out. For each $i=1,2, \ldots, n-m$, let $A_{i}^{*}$ be the multiset of entries that are popped from the $i$ th cell of the top row of $S^{*}$ and let $B_{i}$ be the corresponding set of entries that are crossed out of $T$. One can easily see that $A_{i}^{*}$ is actually a set and $\infty \in A_{i}^{*}$ for all $i$. Now let $A_{i}=A_{i}^{*} \backslash\{\infty\}$. So $\left|A_{i}\right|=\left|B_{i}\right|-1$. It is easily observed that $M_{m, n}$ is an orientable pseudomanifold whenever $m=n-1$ which implies that its top homology is cyclic. For $i=1, \ldots, n-m$, let $\alpha_{i}$ be the generator of the cyclic group $\tilde{H}_{\left|A_{i}\right|-1}\left(M_{A_{i}, B_{i}}\right)$, which is unique up to sign. Now define

$$
\rho(S, T)=\alpha_{1} * \cdots * \alpha_{n-m}
$$

which is unique up to sign.

We demonstrate the procedure for constructing $\rho(S, T)$ on the tableaux $S, T$ of Figure 6.1. First entry 8 is crossed out of $T$ and entry 1 is popped from the first cell of the first row of $S^{*}$. So 1 is placed in $A_{1}^{*}$ and 8 is placed in $B_{1}$. Next entry 7 is crossed out and entry 3 is popped from the second cell. So 3 is placed in $A_{2}^{*}$ and 7 is placed in $B_{2}$. We eventually end up with

$$
A_{1}^{*}=\{1,2, \infty\}, A_{2}^{*}=\{3,4, \infty\}, A_{3}^{*}=A_{4}^{*}=\{\infty\},
$$




$$
B_{1}=\{1,3,8\}, B_{2}=\{2,5,7\}, B_{3}=\{4\}, B_{4}=\{6\} .
$$

Hence

$$
A_{1}=\{1,2\}, A_{2}=\{3,4\}, A_{3}=A_{4}=\emptyset
$$

Now $\rho(S, T)=\alpha_{1} * \alpha_{2}$, where $\alpha_{1}$ is the generator of $\tilde{H}_{1}\left(M_{\{1,2\},\{1,3,8\}}\right)$ and $\alpha_{2}$ is the generator of $\tilde{H}_{1}\left(M_{\{3,4\},\{2,5,7\}}\right)$.

Theorem 6.5 (Shareshian and Wachs [40]). Let $m \leq n$. Then

- $\left\{\rho(S, T) \mid(S, T) \in \mathcal{P}_{m, n}\right\}$ is a basis for $\tilde{H}_{m-1}\left(M_{m, n}\right)$.

- $\left\{\gamma(S, T) \mid(S, T) \in \mathcal{P}_{m, n}\right\}$ is a basis for the free part of $\tilde{H}^{m-1}\left(M_{m, n}\right)$.

Theorem 6.5 is proved by finding an ordering of the pairs of standard tableaux

$$
\left(S_{1}, T_{1}\right), \ldots,\left(S_{t}, T_{t}\right)
$$

in $\mathcal{P}_{m, n}$ such that the matrix

$$
\left(\left\langle\rho\left(S_{i}, T_{i}\right), \gamma\left(S_{j}, T_{j}\right)\right\rangle\right)_{i, j=1, \ldots, t}
$$

is unitriangular. Here $\langle$,$\rangle denotes the pairing of homology and coho-$ mology. The invertibility of the matrix establishes independence of the cycles (and cocycles). The result then follows from the Garst (Corollary 3.3) or Friedman-Hanlon (Theorem 3.2) determination of the rank of rational homology.

6.4. Homology of the $m \times(2 m-2)$ chessboard complex. For $m+n \equiv 1 \bmod 3$, the bottom nonvanishing homology of $M_{m, n}$ is completely determined by Theorem 6.3 when $m \leq n<2 m-2$ and by the Friedman-Hanlon result (Theorem 3.2) when $n>2 m-2$. This leaves $n=2 m-2$ as the only remaining case. To see if $\tilde{H}_{\nu_{m, 2 m-2}}\left(M_{m, 2 m-2}\right)$ is finite, one can apply the Friedman-Hanlon result. In this case $\mathcal{R}(m, 2 m-2, m-1)$ consists of a single pair of partitions; namely the pair $\left((m),(m-1)^{2}\right)$. From this one sees that the bottom nonvanishing homology is not finite and that its free part has rank equal to the number of standard Young tableaux of shape $(m-1)^{2}$ which is well-known to be the Catalan number $c_{m}:=\frac{1}{m}\left(\begin{array}{c}2 m-2 \\ m-1\end{array}\right)$. The next result shows that there is no torsion part. Hence for all values of $m, n$ such that $m+n \equiv 1 \bmod 3$, the bottom nonvanishing homology of $M_{m, n}$ is either $\mathbb{Z}_{3}$ or free.

Theorem 6.6 (Shareshian and Wachs [40]). For $n=2 m-2$, we have

$$
\tilde{H}_{\nu_{m, n}}\left(M_{m, n}\right) \cong \mathbb{Z}^{c_{m}} \text {. }
$$

Consequently for $n \geq 2 m-2$ the homology of the chessboard complex $M_{m, n}$ is free in all degrees. 
According to Theorem 3.2, the representation of $\mathfrak{S}_{2 m-2}$ on $\tilde{H}_{m-2}\left(M_{m, 2 m-2} ; \mathbb{C}\right)$ is isomorphic to the Specht module indexed by the partition $\lambda=(m-1)^{2}$. Theorem 6.6 is proved by constructing an explicit $\mathbb{Z}$-module isomorphism from the integral Specht module $S_{\mathbb{Z}}^{\lambda}$ to $\tilde{H}_{\nu_{m, n}}\left(M_{m, n}\right)$. For a bijective tableau $T$ of shape $\lambda$, i.e., a tableau of shape $\lambda$ with distinct entries in $[|\lambda|]$, let $e_{T}$ be the polytabloid corresponding to $T$ (see (7.1) for the definition of polytabloid). It is wellknown that the set $\left\{e_{T} \mid T\right.$ a bijective tableau of shape $\left.\lambda\right\}$ generates $S_{\mathbb{Z}}^{\lambda}$. For $T$ a tableau of shape $(m-1)^{2}$, let $\rho_{T}$ be the cycle in $\tilde{H}_{m-2}\left(M_{m, 2 m-2}\right)$ given by

$$
\rho_{T}=\alpha_{1} * \alpha_{2} * \cdots * \alpha_{m-1}
$$

where $\alpha_{i}$ is the cycle $\left(i, a_{i}\right)-\left(i, b_{i}\right)$ in $\tilde{H}_{0}\left(M_{\{i\},\left\{a_{i}, b_{i}\right\}}\right)$ and $\left[\begin{array}{c}a_{i} \\ b_{i}\end{array}\right]$ is the $i^{\text {th }}$ column of $T$. It is shown, again using the basis for top homology given in Theorem 6.5, that

$\operatorname{span}\left\{\rho_{T} \mid T\right.$ a bijective tableau of shape $\left.(m-1)^{2}\right\}=\tilde{H}_{m-2}\left(M_{m, 2 m-2}\right)$.

The homomorphism $\psi: S_{\mathbb{Z}}^{\lambda} \rightarrow \tilde{H}_{m-2}\left(M_{m, 2 m-2}\right)$ is defined on generators by

$$
\psi\left(e_{T}\right)=\rho_{T}
$$

It is shown that this map is well-defined by checking that the Garnir relations of $S^{\lambda}$ map to the boundary relations of $\tilde{H}_{m-2}\left(M_{m, 2 m-2}\right)$. Clearly by (6.1), $\psi$ is surjective. It therefore follows from the fact that the modules are isomorphic over the rationals, that the homomorphism $\psi$ is an isomorphism. This argument also yields

Corollary 6.7. The set

$$
\left\{\rho_{T} \mid T \text { a standard tableau of shape }(m-1)^{2}\right\}
$$

is a basis for $\tilde{H}_{m-2}\left(M_{m, 2 m-2}\right)$.

\subsection{Open problems.}

Problem 6.8. Eliminate the use of the computer in showing that $\tilde{H}_{\nu_{12}}\left(M_{12}\right)$ and $\tilde{H}_{\nu_{5,5}}\left(M_{5,5}\right)$ have exponent 3 . This could lead to a proof that $\tilde{H}_{\nu_{9,9}}\left(M_{9,9}\right)$ has exponent 3 and thereby improve Theorem 6.3.

Problem 6.9. Find a formula or some combinatorial expression for the ranks $r_{n}$ and $r_{m, n}$ of Theorems 6.2 and 6.3, respectively. In [40] the following rough bounds on $r_{n}$ are derived for $n \geq 9$ :

- If $n \equiv 0 \bmod 3$ then $n-1 \leq r_{n} \leq 2(n-2) r_{n-3}$. 
- If $n \equiv 2 \bmod 3$ then

$$
(n-1)(n-3)-1 \leq r_{n} \leq(n-2)(n-3)(n-4) r_{n-5}+2(n-2) r_{n-3}
$$

Analogous bounds are also given for the rank $r_{m, n}$ of the bottom nonvanishing homology of the chessboard complex.

Problem 6.10. Determine the bottom nonvanishing homology of the chessboard complex $M_{m, n}$ for $n=2 m-i$, where $i=3,4,6,7,10$. All other values of $i$ are covered by Theorems 3.2, 6.3 and 6.6. Computer computations of Dumas (personal communication) have produced partial results for $(m, n)=(7,8)$ which include the occurrence of 2-torsion. It is shown in [40] that for $(m, n)=(7,8)$, there is 3 -torsion and if the exponent of this 3 -torsion turns out to be 3 then the exponent is 3 in all cases of Theorem 6.3.

Problem 6.11. Determine the torsion parts of arbitrary degree homology groups of the matching and chessboard complexes.

\section{General Degree Bounds}

The bounded degree graph complex for the complete graph is defined as follows. Let $\gamma=\left(\gamma_{1}, \ldots, \gamma_{n}\right)$ be an $n$-tuple of nonnegative integers. The bounded degree graph complex $\Delta_{\gamma}$ is defined to be the simplicial complex of all graphs on node set $[n]$ in which node $i \in[n]$ has degree at most $\gamma_{i}$. Loops are allowed and contribute 2 to the degree of the node. Multiple edges are not allowed.

The bounded degree graph complex for a complete bipartite graph is defined as follows. Let $\gamma=\left(\gamma_{1}, \ldots, \gamma_{m}\right)$ and $\delta=\left(\delta_{1}, \ldots, \delta_{n}\right)$ be sequences of nonnegative integers. The bounded degree bipartite graph complex $\Delta_{\gamma, \delta}$ is defined to be the simplicial complex of all bipartite graphs on partitioned node set $[m] \uplus[n]^{\prime}$ in which node $i \in[m]$ has degree at most $\gamma_{i}$ and node $i^{\prime} \in[n]^{\prime}$ has degree at most $\delta_{i}$. Note that $\Delta_{\gamma, \delta}$ can be viewed as the complex of rook placements on an $m \times n$ chessboard so that there are at most $\gamma_{i}$ rooks in row $i$ for each $i \in[m]$ and at most $\delta_{i}$ rooks in column $i$ for each $i \in[n]$. For this reason $\Delta_{\gamma, \delta}$ has also been called the chessboard complex with multiplicities.

We may assume without loss of generality that $\gamma$ and $\delta$ are weakly decreasing sequences of positive integers (i.e., partitions) since relabeling the nodes and removing nodes with degree bound 0 gives an isomorphic simplicial complex. For $\gamma=j^{a_{j}}(j-1)^{a_{j-1}} \cdots 1^{a_{1}}$, we let $\Sigma(\gamma)$ denote the Young subgroup $\mathfrak{S}_{a_{j}} \times \cdots \times \mathfrak{S}_{a_{1}} \subseteq \mathfrak{S}_{n}$. The Young subgroup $\Sigma(\gamma)$ acts naturally on $\Delta_{\gamma}$ by permuting graph nodes of like degree bound as does the direct product $\Sigma(\gamma) \times \Sigma(\delta)$ act on $\Delta_{\gamma, \delta}$. These actions induce 
representations of $\Sigma(\gamma)$ and $\Sigma(\gamma) \times \Sigma(\delta)$ on homology of $\Delta_{\gamma}$ and $\Delta_{\gamma, \delta}$, respectively.

7.1. Representations on homology. In this subsection we discuss Reiner and Roberts' generalizations of Bouc's decomposition of the homology of the matching complex (Theorem 3.1) and Friedman and Hanlon's decomposition of the homology of the chessboard complex (Theorem 3.2).

Let $\lambda$ and $\gamma$ be partitions of $N$ and let $T$ be a tableau of shape $\lambda$ and content $\gamma$, where the content of a tableau is a sequence whose $i$ th entry is the number of $i$ 's appearing in the tableau. The group $\Sigma(\gamma)$ acts on the left on $T$ by permuting the entries of $T$ that have the same multiplicity. The group $\mathfrak{S}_{N}$ acts on the right on $T$ by permuting the cells of $T$. These actions commute and preserve the shape and content of $T$. The orbit of $T$ under the right action of the subgroup of row stabilizers is called a tabloid and is denoted by $[T]$. Let $C_{\lambda}$ be the subgroup of column stabilizers of $T$. The polytabloid indexed by $T$ is defined to be

$$
e_{T}:=\sum_{\sigma \in C_{\lambda}} \operatorname{sgn} \sigma[T \sigma]
$$

in the $k$-vector space generated by tabloids of shape $\lambda$ and content $\gamma$. The left action of $\Sigma(\gamma)$ on tableaux extends to a left action on polytabloids given by $\alpha e_{T}=e_{\alpha T}$ for $\alpha \in \Sigma(\gamma)$. Let $S^{\lambda}(\gamma)$ be the $\Sigma(\gamma)$-module generated by polytabloids of shape $\lambda$ and content $\gamma$.

Theorem 7.1 (Reiner and Roberts [37]). For all $p \in \mathbb{Z}$ and partitions $\gamma$ and $\delta$, the following module isomorphisms hold

$$
\begin{aligned}
& \tilde{H}_{p-1}\left(\Delta_{\gamma} ; k\right) \cong_{\Sigma(\gamma)} \bigoplus_{\substack{\lambda: \lambda \vdash|\gamma| \\
\lambda=\lambda^{\prime} \\
d(\lambda)=|\lambda|-2 p}} S^{\lambda}(\gamma) . \\
& \tilde{H}_{p-1}\left(\Delta_{\gamma, \delta} ; k\right) \cong_{\Sigma(\gamma) \times \Sigma(\delta)} \bigoplus_{\substack{(\lambda, \mu) \in \mathcal{R}(|\gamma|,|\delta|, p)\\
}} S^{\lambda^{\prime}}(\gamma) \otimes S^{\mu}(\delta) .
\end{aligned}
$$

Since the semistandard polytabloids of shape $\lambda$ and content $\gamma$ form a basis for $S^{\lambda}(\gamma)$ one can express the Betti numbers in terms of the Kostka numbers $K_{\lambda, \gamma}$. 
Corollary 7.2. The reduced Betti numbers satisfy

$$
\begin{gathered}
\tilde{\beta}_{p-1}\left(\Delta_{\gamma}\right)=\sum_{\substack{\lambda: \lambda \vdash|\gamma| \\
\lambda=\lambda^{\prime} \\
d(\lambda)=|\lambda|-2 p}} K_{\lambda, \gamma} . \\
\tilde{\beta}_{p-1}\left(\Delta_{\gamma, \delta}\right)=\sum_{(\lambda, \mu) \in \mathcal{R}(|\gamma|,|\delta|, p)} K_{\lambda^{\prime}, \gamma} K_{\mu, \delta} .
\end{gathered}
$$

Example: For $\gamma=(2,2,2)$ we have

$$
\tilde{\beta}_{p-1}\left(\Delta_{\gamma}\right)=\sum_{\substack{\lambda: \lambda \vdash 6 \\ \lambda=\lambda^{\prime} \\ d(\lambda)=6-2 p}} K_{\lambda, \gamma} .
$$

Since the only self-conjugate partition of 6 is $\lambda=(3,2,1)$ and $d(\lambda)=2$, homology vanishes in all dimensions except $(6-2) / 2-1=1$. In dimension 1 we have

$$
\tilde{\beta}_{1}\left(\Delta_{(2,2,2)}\right)=K_{(3,2,1),(2,2,2)}=2 .
$$

In fact, $\Delta_{(2,2,2)}$ has the homotopy type of a wedge of two 1-spheres.

Since $S^{\lambda}\left(1^{n}\right)=S^{\lambda}$, Bouc's decomposition (Theorem 3.1) and Friedman and Hanlon's decomposition (Theorem 3.2) are special cases of (7.2) and (7.3), respectively. In [31] Karaguezian, Reiner and Wachs show that one can actually derive (7.2) from Bouc's decomposition and (7.3) from Friedman and Hanlon's decomposition. In order to describe this derivation we need to review the notion of weight space of a $G L(V)$ module. See [25] for further information on weight spaces. Let $V$ be a $t$-dimensional vector space over $k$. Fix an ordered basis $v_{1}, \ldots, v_{t}$ of $V$, so that we can view the general linear group $G L(V)$ as a group of $t \times t$ matrices over $k$. For $x_{1}, \ldots, x_{t} \in k$, let $\operatorname{diag}\left(x_{1}, \ldots, x_{t}\right)$ denote the diagonal matrix in $G L(V)$ with these entries. Given a sequence $\gamma=\left(\gamma_{1}, \ldots, \gamma_{t}\right)$ of nonnegative integers, let $x^{\gamma}$ denote the product $x_{1}^{\gamma_{1}} \cdots x_{t}^{\gamma_{t}}$. Given a polynomial representation $U$ of $G L(V)$, the weight space of $U$ corresponding to weight $\gamma$ is denoted by $U_{\gamma}$ and is defined to be

$$
U_{\gamma}=\left\{u \in U: \operatorname{diag}\left(x_{1}, \ldots, x_{t}\right) \cdot u=x^{\gamma} u \quad \forall x_{1}, \ldots, x_{t} \in k\right\} .
$$

For example, if $U=V^{\otimes n}$ then $U_{\gamma}$ is generated by elements of the form $v_{i_{1}} \otimes \cdots \otimes v_{i_{n}}$ where the sequence $i_{1}, \ldots, i_{n}$ has $\gamma_{j}$ occurrences of $j$ for each $j \in[t]$. 
Now suppose $\gamma$ is a partition of length $n$, where $n \leq t$. We shall view $\gamma$ as a weight, i.e., a sequence of $t$ nonnegative integers, by attaching $t-n$ copies of 0 . Then the Young subgroup $\Sigma(\gamma) \hookrightarrow \mathfrak{S}_{n} \hookrightarrow \mathfrak{S}_{t} \hookrightarrow$ $G L(V)$ acts on $U$ and preserves $U_{\gamma}$. So the weight space $U_{\gamma}$ is a $\Sigma(\gamma)$ module. When $\gamma=1^{t}$ one has that $U_{\gamma}$ is an $\mathfrak{S}_{t}$-module.

Recall that the theory of highest weights gives an indexing of irreducible polynomial representations of $G L(V)$ by partitions with at most $t$ parts. Let $V^{\lambda}$ be the irreducible polynomial representation of $G L(V)$ with highest weight $\lambda$ if $\ell(\lambda) \leq \operatorname{dim} V$ and 0 otherwise. By a basic construction of irreducible $G L(V)$-modules (cf. [25, §8.1]), the weight space $V_{\gamma}^{\lambda}$ is isomorphic to the $\Sigma(\gamma)$-module $S^{\lambda}(\gamma)$.

The key idea in deriving (7.2) is the observation that the $\Sigma(\gamma)$ module $\tilde{H}_{p-1}\left(\Delta_{\gamma} ; k\right)$ is the $\gamma$-weight space of a certain polynomial $G L(V)$ module $\mathcal{M}$ described below. The decomposition of the $1^{t}$-weight space of any polynomial representation of $G L(V)$ into irreducible $\mathfrak{S}_{t}$-modules determines the decomposition of the $G L(V)$-module into irreducibles. Indeed the multiplicity of $V^{\lambda}$ is the same as the multiplicity of $S^{\lambda}$ in the $1^{t}$-weight space. Hence Bouc's decomposition determines a decomposition of $\mathcal{M}$ into irreducible $G L(V)$-modules which in turn determines a decomposition of $\mathcal{M}_{\gamma}=\tilde{H}_{p-1}\left(\Delta_{\gamma} ; k\right)$ into the $\gamma$-weight spaces of irreducible $G L(V)$-modules. This decomposition is precisely what is given in (7.2). An analogous argument holds for (7.3).

The $G L(V)$-module $\mathcal{M}$ is the homology a complex $\left(U_{p}(V), \delta_{p}\right)$ of $G L(V)$-modules which we now define. For each $p \in \mathbb{N}$, form the $G L(V)$ module

$$
U_{p}(V)=\wedge^{p}\left(\operatorname{Sym}^{2} V\right) \otimes \operatorname{Sym}^{*} V,
$$

where $\wedge^{p}$ is the $p$ th exterior power, $\operatorname{Sym}^{p}$ is the $p$ th symmetric power and $\mathrm{Sym}^{*}:=\oplus_{p} \operatorname{Sym}^{p}$ is the symmetric algebra. Let $\delta_{p}: U_{p}(V) \rightarrow$ $U_{p-1}(V)$ be defined on generators by

$$
\begin{gathered}
\delta_{p}\left(v_{1} v_{2} \wedge \cdots \wedge v_{2 p-1} v_{2 p} \otimes u\right) \\
=\sum_{j=1}^{p}(-1)^{j} v_{1} v_{2} \wedge \cdots \wedge \widehat{v}_{2 j-1} \widehat{v}_{2 j} \wedge \cdots \wedge v_{2 p-1} v_{2 p} \otimes u v_{2 j-1} v_{2 j},
\end{gathered}
$$

where^ denotes deletion, $v_{i} \in V$ and $u \in \operatorname{Sym}^{*} V$.

Remark 7.3. The decomposition of the homology of $\left(U_{p}(V), \delta_{p}\right)$, into irreducible $G L(V)$-modules was first stated prior to Bouc's work by Józefiak and Weyman [28] (based on work of Józefiak, Pragacz, and Weyman [27]). The proof outlined here (the Dong-Wachs proof of Theorem 3.1 plus the Karaguezian-Reiner-Wachs lifting technique described above) can be viewed as simpler and more elementary. Józefiak 
and Weyman also observe in [28] that by taking the Young dual of $U_{p}(V)$ (i.e., the map that sends irreducible $V^{\lambda}$ to $V^{\lambda^{\prime}}$ ) and the adjoint of $\delta_{p}$, one transforms the complex $\left(U_{p}(V), \delta_{p}\right)$ into the Koszul complex of the free two-step nilpotent Lie algebra $\bigwedge^{2} V \otimes V$ (whose only nonzero bracket on generators is $[x, y]:=x \wedge y$, where $x, y \in V)$. Hence Bouc's decomposition also yields a decomposition of the homology of the free two-step nilpotent Lie algebra. This decomposition was also derived by Sigg [42] by using the discrete Laplacian for Koszul complexes (cf. [21]).

To derive (7.3) from the Friedman-Hanlon decomposition, let $V_{1}$ and $V_{2}$ be finite dimensional vector spaces. One uses the complex $\left(\wedge^{p}\left(V_{1} \otimes V_{2}\right) \otimes\left(\mathrm{Sym}^{*} V_{1} \otimes \mathrm{Sym}^{*} V_{2}\right), \delta_{p}\right)$ of $G L\left(V_{1}\right) \times G L\left(V_{2}\right)$-modules whose differential

$\delta_{p}: \wedge^{p}\left(V_{1} \otimes V_{2}\right) \otimes\left(\mathrm{Sym}^{*} V_{1} \otimes \mathrm{Sym}^{*} V_{2}\right) \rightarrow \wedge^{p-1}\left(V_{1} \otimes V_{2}\right) \otimes\left(\mathrm{Sym}^{*} V_{1} \otimes \mathrm{Sym}^{*} V_{2}\right)$

is defined on generators by

$$
\begin{gathered}
\delta\left(\left(\left(v_{1,1} \otimes v_{2,1}\right) \wedge \cdots \wedge\left(v_{1, p} \otimes v_{2, p}\right)\right) \otimes\left(u_{1} \otimes u_{2}\right)\right)= \\
\sum_{j=1}^{p}(-1)^{j}\left(\left(v_{1,1} \otimes v_{2,1}\right) \wedge \cdots \wedge\left({\widehat{v_{1, j} \otimes v_{2, j}}}_{2} \wedge \cdots \wedge\left(v_{1, p} \otimes v_{2, p}\right)\right) \otimes\left(u_{1} v_{1, j} \otimes u_{2} v_{2, j}\right),\right.
\end{gathered}
$$

where $v_{i, h} \in V_{i}$ and $u_{i} \in \operatorname{Sym}^{*} V_{i}$. The $\gamma, \delta$-weight space of the $p^{t h}$ homology of this complex is isomorphic to $\tilde{H}_{p-1}\left(\Delta_{\gamma, \delta} ; k\right)$.

7.2. Homotopy. This subsection deals with homotopy results of Dong [19] which are, in a sense, predicted by (7.4). These homotopy results are quite interesting in their own right and yield corresponding results about quadratic Veronese resolutions (cf. Section 2) that are independent of the characteristic of $k$, see [19].

An immediate consequence of (7.4) is that $\Delta_{\gamma}$ is $\mathbb{Q}$-acyclic if and only if for all self-conjugate partitions $\lambda \vdash|\gamma|$, the Kostka number $K_{\lambda, \gamma}=0$. It is well-known and easy to see that $K_{\lambda, \gamma} \neq 0$ if and only if $\gamma \unlhd \lambda$, where $\unlhd$ denotes dominance order. Hence, it follows from (7.4) that $\Delta_{\gamma}$ is $\mathbb{Q}$-acyclic if and only if no self-conjugate partition $\lambda \vdash|\gamma|$ dominates $\gamma$. Dong obtains the following homotopy version of this result by using techniques from Forman's discrete Morse theory [23].

Theorem 7.4 (Dong [19]). For any partition $\gamma$, the following are equivalent

1. $\Delta_{\gamma}$ is $\mathbb{Q}$-acyclic.

2. $\Delta_{\gamma}$ is acyclic.

3. $\Delta_{\gamma}$ is contractible.

4. $\Delta_{\gamma}$ is collapsible. 
5. There is no self-conjugate partition of $|\gamma|$ that dominates $\gamma$.

Corollary 7.5 (Dong [19]). If $\gamma$ is not dominated by its conjugate then $\Delta_{\gamma}$ is contractible.

The converse of Corollary 7.5 does not hold. There are partitions that are dominated by their conjugates but not by any self-conjugate partition of the same size. For example $1^{2}$ is such a partition. In [19] a complete characterization of such partitions is given.

We will say that a partition $\gamma$ is balanced if the number of cells below its diagonal equals the number of cells above its diagonal. It is not hard to see that for balanced $\gamma$, if $K_{\lambda, \gamma} \neq 0$ and $\lambda=\lambda^{\prime}$ then $d(\lambda)=d(\gamma)$. It therefore follows from (7.4) that the rational homology of $\Delta_{\gamma}$ vanishes in all dimensions but dimension $\frac{|\gamma|-d(\gamma)}{2}-1$. Dong uses discrete Morse theory to obtain the following homotopy version of this result.

Theorem 7.6 (Dong [19]). Suppose $\gamma$ is a balanced partition. Then $\Delta_{\gamma}$ has the homotopy type of a wedge of $\left(\frac{|\gamma|-d(\gamma)}{2}-1\right)$-dimensional spheres. The number of spheres is positive if and only if $\gamma$ is dominated by its conjugate.

Dong obtains the same result for partitions for which the difference between the number of cells below the diagonal and the number of cells above the diagonal is 2 . If the difference is 1 or more than 2 , homology can occur in several dimensions. Dong gives $\gamma=3^{2} 1^{3}$ as an example and uses (7.4) to establish homology in several dimensions.

For any partition $\gamma$, let $\mathcal{T}(\gamma)$ be the set of semistandard tableaux of content $\gamma$ and self-conjugate shape. We will write $\gamma=\tilde{\gamma} \circ \hat{\gamma}$ if $\gamma=\left(\alpha_{1}, \ldots, \alpha_{d} \mid \beta_{1}, \ldots, \beta_{d}\right), \tilde{\gamma}=\left(\alpha_{1}, \ldots, \alpha_{l} \mid \beta_{1}, \ldots, \beta_{l}\right)$ and $\hat{\gamma}=$ $\left(\alpha_{l+1}, \ldots, \alpha_{d} \mid \beta_{l+1}, \ldots, \beta_{d}\right)$, where $0 \leq l \leq d$. Dong constructs a bijection between $\mathcal{T}(\tilde{\gamma} \circ \hat{\gamma})$ and $\mathcal{T}(\tilde{\gamma}) \times \mathcal{T}(\hat{\gamma})$ for balanced $\tilde{\gamma}$. It follows from this bijection and (7.4) that the Betti numbers of $\Delta_{\tilde{\gamma} \circ \hat{\gamma}}$ can be expressed in terms of the Betti numbers of $\Delta_{\tilde{\gamma}}$ and $\Delta_{\hat{\gamma}}$. The homotopy version of this relation is given in the following result.

Theorem 7.7 (Dong[19]). Let $\gamma=\tilde{\gamma} \circ \hat{\gamma}$, where $\tilde{\gamma}$ is balanced. Then

$$
\Delta_{\gamma} \simeq \Delta_{\tilde{\gamma}} * \Delta_{\hat{\gamma}}
$$

where $*$ denotes the join operation and $\simeq$ denotes homotopy equivalence. Consequently $\Delta_{\gamma}$ has the homotopy type of a wedge of $m$ copies of the $\frac{|\tilde{\gamma}|-d(\tilde{\gamma})}{2}$-suspension of $\Delta_{\hat{\gamma}}$, where $m=\sum_{\lambda=\lambda^{\prime}} K_{\lambda, \tilde{\gamma}}$.

It can be shown algebraically or combinatorially that

$$
K_{\lambda, \gamma}=K_{N^{N}-\lambda, N^{N}-\gamma}
$$


whenever $N \geq \ell(\gamma), \ell(\lambda), \ell\left(\gamma^{\prime}\right), \ell\left(\lambda^{\prime}\right)$ [43, Ex. 7.41]. By (7.4) this implies a relation between the Betti numbers of $\Delta_{\gamma}$ and $\Delta_{N^{N}-\gamma}$ which suggests a relationship between the Alexander dual of $\Delta_{\gamma}$ and $\Delta_{N^{N}-\gamma}$. The Alexander dual of a simplicial complex $\Delta$ on vertex set $V$ is the simplicial complex

$$
\Delta^{\vee}:=\{F \subseteq V \mid V-F \notin \Delta\} .
$$

Note that if $\gamma_{i} \geq 2$ for all $i$ then $\Delta_{\gamma}^{\vee}$ is the simplicial complex of graphs on $[n]$ such that at least one node $i \in[n]$ has degree less than or equal to $n-\gamma_{i}$.

Theorem 7.8 (Dong [19]). Let $n \geq \gamma_{1} \geq \gamma_{2} \geq \cdots \geq \gamma_{n} \geq 1$. If $\gamma_{n} \geq 2$ then

$$
\Delta_{\gamma}^{\vee} \simeq \operatorname{Susp}^{n-1}\left(\Delta_{n^{n}-\gamma}\right),
$$

where $\mathrm{Susp}^{j}$ denotes the $j$-fold suspension. More generally, if $\gamma_{n-i} \geq 2$ and $\gamma_{n-i+1}=\cdots=\gamma_{n}=1$ then

$$
\operatorname{Susp}^{i}\left(\Delta_{\gamma}^{\vee}\right) \simeq \operatorname{Susp}^{n-1}\left(\Delta_{n^{n}-\gamma}\right) .
$$

The proof makes use of the Wedge Lemma from the theory of diagrams of spaces, cf. [52].

\subsection{Open problems.}

Problem 7.9. Generalize the results of Sections 5 and 6 to bounded degree graph complexes. In particular, find the connectivity degree and determine the torsion in the bottom nonvanishing homology.

Problem 7.10. Dong (personal communication) has obtained a bipartite graph analogue of Theorem 7.8. Find bipartite graph analogues of the other homotopy results in Section 7.2 which are predicted by (7.5).

Problem 7.11. Are there nice bases for homology and cohomology of $\Delta_{\gamma}$ when $\gamma$ is balanced? This basis should be indexed in a natural way by standard tableaux of content $\gamma$ and self-conjugate shape.

\section{Digraphs, MUltigraphS AND $B_{n}$-ANAlogues}

In this section we present results of Wachs [51] extending the work of previous sections to digraph and multigraph complexes. The digraph version of the matching complex considered in [51] is different from one considered earlier by Björner and Welker [10] which is also discussed here. This section also deals with $B_{n}$-analogues and wreath product generalizations of results in previous sections. 
8.1. Matching complexes. When one multiplies the two Littlewood identities (3.3) and (4.1) one obtains

$$
\prod_{i, j}\left(1-x_{i} x_{j}\right) \prod_{i}\left(1-x_{i}\right)^{-1}=\sum_{\substack{\lambda=\lambda^{\prime} \\ \beta \in \mathcal{B}}}(-1)^{(\mid \lambda \otimes \beta) \mid-d(\lambda)) / 2} s_{\lambda \otimes \beta}
$$

where $\mathcal{B}$ is the set of partitions of the form $\left(\alpha_{1}+1, \ldots, \alpha_{d}+1\right.$ $\left.\alpha_{1}, \ldots, \alpha_{d}\right)$ for some $d$, and $\lambda \otimes \beta$ denotes the skew shape

$$
\left(\lambda_{1}+\beta_{1}, \lambda_{2}+\beta_{1}, \ldots, \lambda_{\ell(\lambda)}+\beta_{1}, \beta_{1}, \ldots, \beta_{\ell(\beta)}\right) / \beta_{1}^{\ell(\lambda)} .
$$

By extracting the degree $n$ terms and applying the inverse of the Frobenius characteristic map one gets

$$
\bigoplus_{p \geq 0}(-1)^{p}\left(S^{1^{p}} \circ\left(S^{1} \otimes S^{1}\right)\right) \cdot S^{n-2 p} \cong \bigoplus_{p \geq 0}(-1)^{p} \bigoplus_{\substack{\lambda=\lambda^{\prime} \\ \beta \in \mathcal{B} \\|\lambda|+|\beta|=n \\ d(\lambda)=n-2 p}} S^{\lambda \otimes \beta}
$$

The $p^{\text {th }}$ summand on the left side of this isomorphism is the representation of $\mathfrak{S}_{n}$ on the $(p-1)^{\text {st }}$ chain space of the directed matching complex which is defined to be the simplicial complex of all directed graphs on node set $[n]$ in which each node has total degree (i.e., indegree plus out-degree) at most 1 . So it is reasonable to expect that the inner sum of the right side is the homology of the directed matching complex, making the isomorphism the Hopf trace formula for the directed matching complex. This is indeed the case. Let $M_{n}^{D}$ denote the directed matching complex on node set $[n]$.

Theorem 8.1 (Wachs [51]). For $p, n \in \mathbb{Z}$, where $n \geq 1$,

$$
\tilde{H}_{p-1}\left(M_{n}^{D} ; k\right) \cong \mathfrak{S}_{n} \bigoplus_{\substack{\lambda=\lambda^{\prime} \\ \beta \in \mathcal{B} \\|\lambda|+|\beta|=n \\ d(\lambda)=n-2 p}} S^{\lambda \otimes \beta},
$$

where $\mathcal{B}$ is the set of partitions of the form $\left(\alpha_{1}+1, \ldots, \alpha_{d}+1\right.$ $\left.\alpha_{1}, \ldots, \alpha_{d}\right)$ for some $d$.

The directed matching complex can be viewed as the matching complex of a multigraph. For $r, n \geq 1$, let $K_{n}^{r}$ be the complete $r$-multigraph on node set $[n]$, i.e., the multigraph with $r$ distinct edges between each (unordered) pair of nodes in node set $[n]$. Let $M_{n}^{r}$ be the simplicial complex of all subgraphs of $K_{n}^{r}$ for which each node has degree at most 
1. We will think of the $r$ distinct edges as colored edges and denote the edge between node $i$ and node $j$ colored by $c \in[r]$ as $(\{i, j\}, c)$. Let $\mathfrak{S}_{n}$ act on $M_{n}^{r}$ by permuting node labels. The permutation $\sigma \in \mathfrak{S}_{n}$ sends colored edge $(\{i, j\}, c)$ to $(\{\sigma(i), \sigma(j)\}, c)$. There is an obvious isomorphism between simplicial complexes $M_{n}^{D}$ and $M_{n}^{2}$. However, this isomorphism is not $\mathfrak{S}_{n}$-equivariant. For example transposition $(1,2)$ sends directed edge $(1,2)$ to directed edge $(2,1)$. This translates under the isomorphism to changing the color of the colored edge $(\{1,2\}, c)$. The following generalization of Theorem 3.1 shows that the $\mathfrak{S}_{n}$-homology modules of $M_{n}^{D}$ and $M_{n}^{2}$ are related by a sign twist.

Theorem 8.2 (Wachs [51]). For $p, n, r \in \mathbb{Z}$, where $n, r \geq 1$,

$$
\tilde{H}_{p-1}\left(M_{n}^{r} ; k\right) \cong \mathfrak{S}_{n} \bigoplus_{\substack{\lambda=\lambda^{\prime} \\ \beta \in \mathcal{B} \\|\lambda|+|\beta|=n \\ d(\lambda)=n-2 p}}(r-1)^{|\beta| / 2} S^{\lambda \otimes \beta^{\prime}}
$$

The proofs of Theorems 8.1 and 8.2 rely on the following construction. Let $\Delta$ be a simplicial complex on vertex set $V$ and let $r \geq 1$. We form a new simplicial complex $\Delta^{r}$, called the $r$-inflation of $\Delta$. The vertex set of $\Delta^{r}$ is $\{(v, c) \mid v \in V, c \in[r]\}$ and the faces of $\Delta^{r}$ are of the form $\left\{\left(v_{1}, c_{1}\right), \ldots,\left(v_{k}, c_{k}\right)\right\}$ where $\left\{v_{1}, \ldots, v_{k}\right\}$ is a $k$ element face of $\Delta$ and $c_{j} \in[r]$ for all $j=1, \ldots, k$. We can think of $\left\{\left(v_{1}, c_{1}\right), \ldots,\left(v_{k}, c_{k}\right)\right\}$ as a coloring of the vertices of face $\left\{v_{1}, \ldots, v_{k}\right\}$. It is easy to see that the multigraph matching complex $M_{n}^{r}$ is the $r$-inflation of the matching complex $M_{n}$.

Let $\bigvee$ denote the wedge operation on connected topological spaces. For any connected topological space $X$, let $X^{\vee j}$ denote the wedge of $j$ copies of $X$. For any simplicial complex $\Delta$, let and let $\operatorname{lk}_{\Delta} F$ denote the link of a face $F$ in $\Delta$.

Theorem 8.3 (Björner, Wachs, Welker [7]). For any connected simplicial complex $\Delta$ and $r \geq 1$,

$$
\Delta^{r} \simeq \bigvee_{F \in \Delta} \operatorname{Susp}^{|F|}\left(\mathrm{lk}_{\Delta} F\right)^{\vee\left((r-1)^{|F|}\right)} .
$$

Note that if $\Delta$ is not connected then one can apply Theorem 8.3 to each component of $\Delta$. Clearly $M_{n}$ is connected if and only if $n=2$ or $n \geq 5$.

Corollary 8.4 (Wachs[51]). For $r \geq 1$ and $n \geq 5$,

$$
M_{n}^{r} \simeq \bigvee_{i=0}^{\left\lfloor\frac{n}{2}\right\rfloor} \operatorname{Susp}^{i}\left(M_{n-2 i}\right)^{\vee a(n, r, i)}
$$


where $a(n, r, i)=(r-1)^{i} \frac{n !}{(n-2 i) ! 2^{i} i !}$.

In [4], Björner uses the theory of subspace arrangements to prove the $r=2$ case of Theorem 8.3. The general result is a consequence of a generalization of Quillen's fiber lemma due to Björner, Wachs and Welker [7]. An equivariant homology version of Theorem 8.3 is used to prove Theorems 8.1 and 8.2.

It follows from Corollary 8.4 that all the homotopy and integral homology results for the matching complex given in Sections 5 and 6 can be lifted to the multigraph matching complex. We have the following result, for example.

Theorem 8.5 (Wachs [51]). For $r \geq 1$ and $n \geq 2$, the connectivity degree of the multigraph matching complex $M_{n}^{r}$ is $\nu_{n}-1$. Moreover, if $n \equiv 1 \bmod 3$ then

$$
\tilde{H}_{\nu_{n}}\left(M_{n}^{r}\right)=\tilde{H}_{\nu_{n}}\left(M_{n}\right)
$$

and if $n \equiv 0,2 \bmod 3$ then

$$
\tilde{H}_{\nu_{n}}\left(M_{n}^{r}\right)=\tilde{H}_{\nu_{n}}\left(M_{n}\right) \oplus(r-1)\left(\begin{array}{l}
n \\
2
\end{array}\right) \tilde{H}_{\nu_{n-2}}\left(M_{n-2}\right) .
$$

Now let us turn to the multigraph version of the chessboard complex; that is, the matching complex of a complete bipartite $r$-multigraph. Such a complex can be viewed as the simplicial complex of $r$-colored rook placements on an $m \times n$ chessboard. An $r$-colored rook placement is a placement of colored rooks on an $m \times n$ chessboard, such that each row and each column gets at most one colored rook whose color is in $[r]$. Note that this complex is simply the the $r$-inflation of the chessboard complex $M_{m, n}$. We therefore denote the complex by $M_{m, n}^{r}$.

The multigraph version of the chessboard complex was introduced by Garst [26] as a class of Tits coset complexes that generalizes the chessboard complexes. Recall that the definition of Tits coset complex given in Section 2 involves a group $G$ and subgroups $G_{1}, \ldots, G_{m}$. Garst sets $G$ equal to the wreath product $\mathfrak{S}_{n} \imath C_{r}$ where $C_{r}$ is the cyclic group of order $r$. Elements of $\mathfrak{S}_{n} \imath C_{r}$ can be viewed as "colored permutations", i.e., words $w=w_{1} w_{2} \cdots w_{n}$ where for each $i$, the letter $w_{i}=\left(\sigma_{i}, c_{i}\right) \in$ $[n] \times C_{r}$ and the $\sigma_{i}$ are distinct. For each $i=1,2, \ldots, m$, set

$$
G_{i}=\left\{w \in \mathfrak{S}_{n} \prec C_{r} \mid w_{i}=(i, e)\right\},
$$

where $e$ is the identity of $C_{r}$. It is easy to see that $\Delta\left(G ; G_{1}, \ldots, G_{m}\right)$ is $M_{m, n}^{r}$. Since $\mathfrak{S}_{n} \prec C_{2}$ is the Weyl group $B_{n}$, the bicolored chessboard complex $M_{m, n}^{2}$ can be viewed as a $B_{n}$-analogue of the chessboard complex. 
Garst proves that $M_{m, n}^{r}$ is Cohen-Macaulay if and only if $2 m-1 \leq n$. He also generalizes Corollary 3.3 by decomposing the representation of $\mathfrak{S}_{n} \prec C_{r}$ on the top homology of $M_{m, n}^{r}$ into irreducibles. The first result turns out to be an immediate consequence of the fact that the inflation of a Cohen-Macaulay simplicial complex is Cohen-Macaulay. This fact follows from Theorem 8.3 and is proved in [7]. The second result is generalized by Wachs [51] to arbitrary degree homology by using an equivariant homology version of Theorem 8.3. We state this result just for $r=2$ below.

The direct product $B_{m} \times B_{n}$ acts on $M_{m, n}^{2}$ in the following way. Let $((a, b), c)$ be a vertex of $M_{m, n}^{2}$, i.e., a placement of a rook of color $c$ in row $a$ and column $b$, where $a \in[m], b \in[n]$ and $c= \pm 1$. Let $\sigma \in \mathfrak{S}_{m}$, $\tau \in \mathfrak{S}_{n}, \epsilon \in\{-1,+1\}^{m}$ and $\delta \in\{-1,+1\}^{n}$. We view $(\sigma, \epsilon)$ as an element of $B_{m}$ and $(\tau, \delta)$ as an element of $B_{n}$. The action of $B_{m}$ on $M_{m, n}^{2}$ is given by letting $(\sigma, \epsilon)$ take $((a, b), c)$ to $\left((\sigma(a), b), \epsilon_{a} c\right)$ and the action of $B_{n}$ is given by letting $(\tau, \delta)$ take $((a, b), c)$ to $\left((a, \tau(b)), \delta_{b} c\right)$. These actions clearly commute and determine an action of $B_{m} \times B_{n}$ on $M_{m, n}^{2}$.

Recall that the irreducible representations $S^{(\lambda, \beta)}$ of $B_{n}$ are indexed by pairs of partitions $(\lambda, \beta)$ such that $|\lambda|+|\beta|=n$.

Theorem 8.6 (Wachs [51]). For $p, m, n \in \mathbb{Z}$, where $m, n \geq 1$, the following $\left(B_{m} \times B_{n}\right)$-module isomorphism holds

$$
\tilde{H}_{p-1}\left(M_{m, n}^{2} ; k\right) \cong{ }_{B_{m} \times B_{n}} \bigoplus_{\substack{\beta:|\beta| \leq p \\(\lambda, \mu) \in \mathcal{R}(m-|\beta|, n-|\beta|, p-|\beta|)}} S^{\left(\lambda^{\prime}, \beta^{\prime}\right)} \otimes S^{(\mu, \beta)} .
$$

A matching complex version of this result is also given in [51]. A different $B_{n}$-analogue of the matching complex is considered by Shareshian (personal communication).

Theorem 8.3 and its equivariant homology version also provide chessboard complex analogues of all the matching complex theorems of this subsection. The analogue of Theorem 8.2 is a special case of Theorem 8.13 below. It is also obtained by restricting the action of $B_{m} \times B_{n}$ to $S_{m} \times S_{n}$ in the isomorphism of Theorem 8.6. We now state the analogues of Theorems 8.4 and 8.5.

Theorem 8.7 (Wachs [51]). For $m \geq 2, n \geq 3$ and $r \geq 1$,

$$
M_{m, n}^{r} \simeq \bigvee_{i=0}^{m} \operatorname{Susp}^{i}\left(M_{m-i, n-i}\right)^{\vee a(m, n, r, i)},
$$

where $a(m, n, r, i)=(r-1)^{i}\left(\begin{array}{c}m \\ i\end{array}\right)\left(\begin{array}{c}n \\ i\end{array}\right) i !$ 
Theorem 8.8 (Wachs [51]). For $1 \leq m \leq n$ and $1 \leq r$, the connectivity degree of $M_{m, n}^{r}$ is $\nu_{m, n}-1$. Moreover, if $m+n \equiv 1 \bmod 3$ then

$$
\tilde{H}_{\nu_{m, n}}\left(M_{m, n}^{r}\right)=\tilde{H}_{\nu_{m, n}}\left(M_{m, n}\right)
$$

and if $m+n \equiv 0,2 \bmod 3$ then

$$
\tilde{H}_{\nu_{m, n}}\left(M_{m, n}^{r}\right)=\tilde{H}_{\nu_{m, n}}\left(M_{m, n}\right) \oplus(r-1)\left(\begin{array}{c}
m+n \\
2
\end{array}\right) \tilde{H}_{\nu_{m-1, n-1}}\left(M_{m-1, n-1}\right) .
$$

A different type of directed graph matching complex is described in a paper of Björner and Welker [10]. This is the complex of all directed graphs on node set $[n]$ for which the indegree and the outdegree of each node is at most 1. If loops are allowed then this complex is simply the chessboard complex $M_{n, n}$. If loops are not allowed then this complex is the chessboard complex on an $n \times n$ board minus the diagonal. We denote this complex by $D M_{n}$.

Theorem 8.9 (Björner and Welker [10]). The connectivity degree of $D M_{n}$ is at least $\nu_{2 n}-1$.

This is proved by applying a result of Ziegler [53] on shellability of skeleta of nonrectangular shaped chessboard complexes. Integral homology of $D M_{n}$ is computed for $2 \leq n \leq 7$ in [10]. The bound $\nu_{2 n}-1$ is sharp for these values and 3-torsion is the only torsion that occurs.

Conjecture 8.10. The connectivity degree of $D M_{n}$ is $\nu_{2 n}-1 .^{1}$ Moreover, there is some $N$ such that $\tilde{H}_{\nu_{2 n}}\left(D M_{n}\right)$ is a nontrivial 3-group of exponent 3 for all $n \geq N$.

8.2. Bounded degree. For $\gamma$ a partition of length $n$, let $\Delta_{\gamma}^{D}$ denote the complex of digraphs on node set $[n]$ for which the total degree of node $i$ is at most $\gamma_{i}$ for each $i$. Again loops are allowed and contribute 2 to the total degree of a node. Also two edges between a pair of vertices which have opposite directions are allowed. The weight-space technique of Karaguezian, Reiner and Wachs [31] described in Section 7 is used to obtain the following generalization of Theorem 8.1.

Theorem 8.11 (Wachs [51]). For $p \in \mathbb{Z}$ and any partition $\gamma$,

$$
\tilde{H}_{p-1}\left(\Delta_{\gamma}^{D} ; k\right) \cong_{\Sigma(\gamma)} \bigoplus_{\substack{\lambda=\lambda^{\prime} \\ \beta \in \mathcal{B} \\|\lambda|+|\beta|=|\gamma| \\ \\ d(\lambda)=|\gamma|-2 p}} S^{\lambda \otimes \beta}(\gamma),
$$

\footnotetext{
${ }^{1}$ See New Developments Section at the end of the paper.
} 
where $\mathcal{B}$ is the set of partitions of the form $\left(\alpha_{1}+1, \ldots, \alpha_{d}+1\right.$ $\left.\alpha_{1}, \ldots, \alpha_{d}\right)$ for some $d$.

For $r, n \geq 1$, let $K_{n}^{r}$ be the complete $r$-multigraph on node set $[n]$. (We require $K_{n}^{r}$ to have $r$ distinct loops at each node as well as $r$ distinct edges between each pair of nodes.) For any increasing $n$-tuple of positive integers $\gamma$, let the bounded degree multigraph complex $\Delta_{r, \gamma}$ be the simplicial complex of submultigraphs of $K_{n}^{r}$ such that the degree of node $i$ is at most $\gamma_{i}$ for each $i$. (Again loops contribute 2 to the degree of a node.) Note that $\Delta_{r, \gamma}$ is not the $r$-inflation of $\Delta_{\gamma}$. Rather, the $r$ inflation of $\Delta_{\gamma}$ is simplicial complex of subgraphs (not submultigraphs) of $K_{n}^{r}$ with degree bounds $\gamma$. Also note that $\Delta_{2, \gamma}$ is not the bounded degree digraph complex $\Delta_{\gamma}^{D}$ since only one loop is allowed at each node in a digraph of $\Delta_{\gamma}^{D}$ while two loops are allowed in a multigraph of $\Delta_{2, \gamma}$. Again the weight-space technique of Karaguezian, Reiner and Wachs is used to generalize Theorem 8.2 and (7.2).

Theorem 8.12 (Wachs [51]). For $p, r \in \mathbb{Z}$, where $r \geq 1$, and any partition $\gamma$,

$$
\tilde{H}_{p-1}\left(\Delta_{r, \gamma} ; k\right) \cong{ }_{\Sigma(\gamma)} \bigoplus_{\substack{\lambda=\lambda^{\prime} \\ \beta \in \mathcal{B} \\|\lambda|+|\beta|=|\gamma| \\ d(\lambda)=|\gamma|-2 p}}(r-1)^{|\beta| / 2} S^{\lambda \otimes \beta^{\prime}}(\gamma)
$$

Let $r, m, n \geq 1, \gamma$ be a partition of length $m$ and $\delta$ be a partition of length $n$. An $r$-colored $(\gamma, \delta)$-rook placement is a placement of colored rooks on an $m \times n$ chessboard, where row $i$ gets at most $\gamma_{i}$ rooks and column $j$ gets at most $\delta_{j}$ rooks and rooks placed in the same square have distinct colors in $[r]$. Let $\Delta_{r, \gamma, \delta}$ be the complex of $r$-colored $(\gamma, \delta)$ rook placements. This complex can be viewed as a bounded degree bipartite multigraph complex. When $r=2$ the complex can be viewed as a bounded degree directed bipartite graph complex. The following analogue of Theorem 8.12 generalizes (7.3).

Theorem 8.13 (Wachs [51]). For $p, r \in \mathbb{Z}$, where $r \geq 1$, and partitions $\gamma$ and $\delta$, the following $\Sigma(\gamma) \times \Sigma(\delta)$-module isomorphism holds

$$
H_{p-1}\left(\Delta_{r, \gamma, \delta} ; k\right) \cong \bigoplus_{\substack{\beta:|\beta| \leq p \\(\lambda, \mu) \in \mathcal{R}(|\gamma|-|\beta|,|\delta|-|\beta|, p-|\beta|)}}(r-1)^{|\beta|} S^{\lambda^{\prime} \otimes \beta^{\prime}}(\gamma) \otimes S^{\mu \otimes \beta}(\delta) .
$$

\subsection{Open problems.}


Problem 8.14. Can any of Dong's homotopy results (Section 7) for the bounded degree graph complexes be extended to multigraph complexes?

Problem 8.15. Determine the connectivity degree and torsion for the bounded degree digraph and multigraph complexes.

Problem 8.16. Decompose the representation of $\mathfrak{S}_{n}$ on $\tilde{H}_{p}\left(D M_{n} ; k\right)$ into irreducibles and determine the Betti numbers. Also determine the connectivity degree ${ }^{1}$ and torsion for $D M_{n}$, i.e., settle Conjecture 8.10.

Problem 8.17. Consider the bounded degree generalization of $D M_{n}$. This is the complex of all digraphs on node set $n$ for which the indegree of node $i$ is bounded from above by $\gamma_{i}$ and the outdegree of node $i$ is bounded by $\delta_{i}$. If loops are allowed this is the same as $\Delta_{\gamma, \delta}$. If loops are not allowed this is the same as the complex of rook placements on an $n \times n$ board minus the diagonal, in which there are at most $\gamma_{i}$ rooks in row $i$ and at most $\delta_{i}$ rooks in column $i$. What can one say about this complex?

\section{HYPERGRAPHS}

Very few of the results of the previous sections have been generalized to hypergraph complexes. We discuss the generalizations that have been obtained in this section.

Let $2 \leq r \leq n$. An $r$-hypergraph on node set $[n]$ is a set of multisets on $[n]$ containing $r$ elements (counting multiplicity). The multisets are called edges. The degree of a node $i$ is the total number of occurrences of $i$ in the edges of the hypergraph. For $\gamma$ a partition of length $n$, the $\gamma$-bounded degree $r$-hypergraph complex $\Delta_{\gamma}(r)$ is defined to be the simplicial complex of $r$-hypergraphs on $[n]$ for which each node $i$ has degree at most $\gamma_{i}$. When $r=2, \Delta_{\gamma}(r)$ is the bounded degree graph complex $\Delta_{\gamma}$.

When $\gamma=1^{n}, \Delta_{\gamma}(r)$ is the $r$-hypergraph matching complex on node set $[n]$, which we denote by $M_{n}(r)$. The $r$-hypergraph matching complex was first studied by Björner, Lovász, Vrećica and Živaljević [6]. This is the $\mathfrak{S}_{n}$-simplicial complex whose faces are set partitions of subsets of $[n]$ into blocks of size $r$.

For $t \geq 2, i=1, \ldots, t$ and $n_{i} \geq 1$, let $\gamma^{(i)}=\left(\gamma_{1}^{(i)}, \ldots, \gamma_{n_{i}}^{(i)}\right)$ be a partition of length $n_{i}$ and let $\gamma=\left(\gamma^{(1)}, \ldots, \gamma^{(t)}\right)$. A $\gamma$-rook configuration on an $n_{1} \times \cdots \times n_{t}$ chessboard is a subset $A$ of $\left[n_{1}\right] \times \cdots \times\left[n_{t}\right]$ such that for $i=1, \ldots, t$, each $j \in\left[n_{i}\right]$ appears as the $i$ th coordinate of at

\footnotetext{
${ }^{1}$ See New Developments Section at the end of the paper.
} 
most $\gamma_{j}^{(i)}$ elements of $A$. The collection of $\gamma$-rook configurations on an $n_{1} \times \cdots \times n_{t}$ chessboard forms a simplicial complex $\Delta_{\gamma}$ that we call the $\gamma$-chessboard complex. When $\gamma_{j}^{(i)}=1$ for all $i$ and $j \in\left[n_{i}\right]$, the complex $\Delta_{\gamma}$ is a chessboard complex on a $t$-dimensional board which we denote by $M_{n_{1}, \ldots, n_{t}}$. Note that $\Delta_{\gamma}$ can be viewed as the simplicial complex of all sub-t-hypergraphs of the complete t-partite t-hypergraph $K_{n_{1}, \ldots, n_{t}}$ on node set $\left[n_{1}\right] \uplus \cdots \uplus \cup\left[n_{t}\right]$, in which each node $j$ of part $i$ has degree at most $\gamma_{j}^{(i)}$.

Theorem 9.1 (Björner, Lovász, Vrećica and Z̆ivaljević [6]). For $2 \leq$ $r \leq n$, let $\nu_{n}(r)=\left\lfloor\frac{n-2}{2 r-1}\right\rfloor$. For $t$-tuple $\left(n_{1}, \ldots, n_{t}\right)$ of positive integers, where $t \geq 2$, let

$\nu_{n_{1}, \ldots, n_{t}}=\min \left\{n_{1}^{\prime}-1,\left\lfloor\frac{n_{1}^{\prime}+n_{2}^{\prime}-2}{3}\right\rfloor, \ldots,\left\lfloor\frac{n_{1}^{\prime}+n_{2}^{\prime}+\cdots+n_{t}^{\prime}-t}{2 t-1}\right\rfloor\right\}$, where $\left(n_{1}^{\prime}, \ldots, n_{t}^{\prime}\right)$ is a weakly increasing rearrangement of $\left(n_{1}, \ldots, n_{t}\right)$. The connectivity degree of $M_{n}(r)$ is at least $\nu_{n}(r)-1$ and the $\nu_{n}(r)$ skeleton of $M_{n}(r)$ is homotopy Cohen-Macaulay. The connectivity degree of $M_{n_{1}, \ldots, n_{t}}$ is at least $\nu_{n_{1}, \ldots, n_{t}}-1$ and the $\nu_{n_{1}, \ldots, n_{t}}$-skeleton of $M_{n_{1}, \ldots, n_{t}}$ is homotopy Cohen-Macaulay.

It is conjectured in [6] that the bounds in Theorem 9.1 are sharp and that the respective skeleta are shellable. A recent result of Ksontini [33] provides counterexamples to the bound for $M_{n}(r)$. The smallest counterexample is given by $M_{11}(3)$ which has connectivity degree 1 rather than $\nu_{11}(3)-1=0$. Ksontini derives an improved bound which is given in the following theorem.

Theorem 9.2 (Ksontini [33]). Let $\nu_{n}^{\prime}(r)=\left\lfloor\frac{n+r-4}{2 r-1}\right\rfloor$. If $r$ is prime and $n \geq 3 r+2$ then the connectivity degree of $M_{n}(r)$ is at least $\nu_{n}^{\prime}(r)-1$.

Note that since $\nu_{n}^{\prime}(2)=\nu_{n}(2)$, by Theorem 5.2 Ksontini's bound is sharp for $r=2$. Ksontini uses a relationship between $M_{n}(r)$ and the poset of elementary abelian $r$-subgroups of $\mathfrak{S}_{n}$ to prove his result. It is for this reason that $r$ is required to be prime. It seems likely that this condition is not necessary. The following result does not appear in [33], but follows easily from Theorem 9.2.

Corollary 9.3. For $r$ prime and $n \geq 3 r+2$, the $\nu_{n}^{\prime}(r)$-skeleton of $M_{n}(r)$ is homotopy Cohen-Macaulay.

The weight space technique of Section 7 is used in [31] to lift the rational homology version of Theorem 9.1 to bounded degree hypergraph complexes. 
Theorem 9.4 (Karaguezian, Reiner and Wachs [31]). The bounded degree $r$-hypergraph complex $\Delta_{\gamma}(r)$ is $\left(\nu_{|\gamma|}(r)-1\right)$-acyclic over the rationals. The $\gamma$-chessboard complex $\Delta_{\gamma}$ is $\left(\nu_{|\gamma(1)|, \ldots,|\gamma(t)|}-1\right)$-acyclic over the rationals.

Corollary 9.5. The $\nu_{|\gamma|}(r)$-skeleton of the bounded degree $r$-hypergraph complex $\Delta_{\gamma}(r)$ is Cohen-Macaulay over the rationals. The $\nu_{|\gamma(1)|, \ldots,|\gamma(t)|^{-}}$ skeleton of the $\gamma$-chessboard complex $\Delta_{\gamma}$ is Cohen-Macaulay over the rationals.

The acyclicity bound for $\Delta_{\gamma}(r)$ given in Theorem 9.4 can now be improved because of Theorem 9.2.

Björner and Eriksson [5] generalize and unify connectivity results for the matching complex and chessboard complex by introducing a simplicial complex called a packing complex. Karaguezian, Reiner and Wachs [31] introduce a bounded degree version of the packing complex which yields a generalization and unification of the results of Theorem 9.4 and Corollary 9.5.

Much more is known about the top than the bottom nonvanishing homology of the hypergraph matching complex.

Theorem 9.6 (Ksontini [Proposition 6.2] [33]). For $2 \leq r<n$, the top nonvanishing integral homology of $M_{n}(r)$ occurs in degree $\left\lfloor\frac{n-1}{r}\right\rfloor-1$.

Remark 9.7. In [33] it is assumed that $r$ is prime. However the result is obtained in [41] without this assumption.

Theorem 9.6 is used in [33] to show that the degree of the top nonvanishing homology of the Brown complex $\mathcal{S}_{p}\left(\mathfrak{S}_{n}\right)$ (the order complex of the poset of nontrivial $p$-subgroups of $\mathfrak{S}_{n}$ ) and the Quillen complex $\mathcal{A}_{p}\left(\mathfrak{S}_{n}\right)$ (the order complex of the poset of nontrivial elementary abelian $p$-subgroups of $\left.\mathfrak{S}_{n}\right)$ is $\left\lfloor\frac{n}{p}\right\rfloor-1$ for all $3 \leq p \leq n-1$, except for the case that $p=3$ and $n \equiv 0 \bmod 3$. In this case the degree is $\frac{n}{3}-2$. Shareshian and Wachs [41] derive an explicit formula expressing the representation of the symmetric group on the top homology of $\mathcal{S}_{p}\left(\mathfrak{S}_{n}\right)$ and $\mathcal{A}_{p}\left(\mathfrak{S}_{n}\right)$ in terms of the representations of the symmetric groups on the top homology of the $M_{i}(p)$. For $p=3$, it is shown that these $\mathfrak{S}_{n}$-modules are isomorphic to the top homology of $M_{n}(p)$.

When $r=3$ and $n \equiv 1 \bmod 3$, the representation of $\mathfrak{S}_{n}$ on the top homology has the following explicit decomposition into irreducibles.

Theorem 9.8 (Shareshian and Wachs [41]). For $m \geq 1$,

$$
\tilde{H}_{m-1}\left(M_{3 m+1}(3) ; k\right) \cong \mathfrak{S}_{3 m+1} \bigoplus_{\lambda \in \Omega_{m, 3 m+1}} S^{\lambda}
$$


where

$\Omega_{m, 3 m+1}=\left\{\left(\lambda_{1} \geq \cdots \geq \lambda_{m} \geq 1\right) \vdash 3 m+1 \mid \lambda_{i}\right.$ is odd for all $\left.i=1, \ldots, m\right\}$.

The proof of Theorem 9.8 involves symmetric function theory and a long exact sequence of Ksontini [33].

Again the weight space technique [31, Theorem 4.3] can be used to lift these results to the general bounded degree hypergraph complexes.

Corollary 9.9 (Shareshian and Wachs [41]). For $r \geq 2$ and any partition $\gamma$ such that $|\gamma|>r$, the top nonvanishing homology of $\Delta_{\gamma}(r)$ has degree $\left\lfloor\frac{|\gamma|-1}{r}\right\rfloor-1$. When $r=3$ and $|\gamma|=3 m+1$ for some $m \geq 1$, we have

$$
\tilde{H}_{m-1}\left(\Delta_{\gamma}(3) ; k\right) \cong_{\Sigma(\gamma)} \bigoplus_{\lambda \in \Omega_{m, 3 m+1}} S^{\lambda}(\gamma)
$$

\subsection{Open problems.}

Problem 9.10. Is Ksontini's bound $\nu_{n}^{\prime}(r)-1$ for the connectivity degree of the hypergraph matching complex $M_{n}(r)$ sharp? ${ }^{1}$ Find an analogue of Ksontini's bound for the multidimensional chessboard complex. ${ }^{1}$ Do the same for the packing complex of Björner and Eriksson. Find the connectivity degree of the bounded degree hypergraph complexes.

Problem 9.11. Show that the $\nu_{n}^{\prime}(r)$-skeleton of $M_{n}(r)$ is shellable. ${ }^{1}$ Do the same for the appropriate skeleton of the higher dimensional chessboard complex. ${ }^{1}$. Show that the $\nu_{|\gamma|}^{\prime}(r)$-skeleton of $\Delta_{\gamma}(r)$ is shellable and show the analogous skeleton of the higher dimensional chessboard complex is shellable.

Problem 9.12. Generalize the decomposition results of Section 3 and the torsion results of Section 6 to hypergraph complexes.

Problem 9.13. Find chessboard complex analogues of Theorems 9.6 and 9.8 .

\section{ACKNOWLEDGEMENTS}

The author would like to thank her coauthors, Xun Dong, Dikran Karaguezian, Victor Reiner, and John Shareshian, for their collaborations on the papers presented in this survey. She is also grateful to John Shareshian for many valuable comments on a preliminary version of this paper. Part of this paper was written while the author was a member of the Isaac Newton Institute in Cambridge. The author would like to thank the institute for its hospitality and support.

\footnotetext{
${ }^{1}$ See New Developments Section at the end of the paper.
} 


\section{NeW DEVELOPMENTS}

Since this paper was first circulated, a few of the problems posed have been solved.

(1) Athanasiadis [1] has shown that the $\nu_{n}$-skeleton of the matching complex $M_{n}$ is vertex decomposable (Problem 5.4). His vertex decomposition generalizes to a vertex decomposition of the $\nu_{n}^{\prime \prime}(r)$-skeleton of the hypergraph matching complex $M_{n}(r)$, where $\nu_{n}^{\prime \prime}(r)=\left\lfloor\frac{n-r}{r+1}\right\rfloor$. Not only does this imply that the $\nu_{n}^{\prime \prime}(r)$-skeleton (as well as the $\nu_{n}^{\prime}(r)$ skeleton) is shellable (Problem 9.11), it improves Ksontini's bound for the connectivity degree of $M_{n}(r)$ to $\nu_{n}^{\prime \prime}(r)-1$ (Problem 9.10).

Athanasiadis also uses vertex decomposability to show that the $t$ dimensional chessboard complex $M_{n_{1}, \ldots, n_{t}}$ is $\left(\nu_{n_{1}^{\prime}, n_{2}^{\prime}}-1\right)$-connected (Problem 9.10) improving the Björner-Lovász-Vrećica-Zivaljević bound, and that its $\nu_{n_{1}^{\prime}, n_{2}^{\prime}}$-skeleton is shellable (Problem 9.11). He then uses Theorem 5.2 to show that this connectivity bound is sharp.

Athanasiadis' new connectivity bounds can be used to improve Theorem 9.4 and Corollary 9.5.

(2) Shareshian and Wachs [40] have settled one of the conjectures in Conjecture 8.10 (Problem 8.16). They have shown that $\tilde{H}_{\nu_{2 n}}\left(D M_{n}\right) \neq$ 0 , thereby establishing sharpness of the Björner-Welker connectivity bound. The idea is to view $D M_{n}$ as a subcomplex of the chessboard complex $M_{n, n}$ and show that every cycle in a certain generating set for

$\tilde{H}_{\nu_{n, n}}\left(M_{n, n}\right)$, constructed in the proof of Theorem 6.3 , can be realized as a cycle of $\tilde{H}_{\nu_{2 n}}\left(D M_{n}\right)$.

\section{REFERENCES}

[1] C. Athanasiadis, Decompositions and connectivity of matching and chessboard complexes, preprint 2002.

[2] E. Babson, A. Björner, S. Linusson, J. Shareshian and V. Welker, Complexes of not i-connected graphs, Topology 38 (1999), 271-299.

[3] E. Babson and V. Reiner, Coxeter-like complexes, preprint 2001.

[4] A. Björner, Subspace arrangements, First European Congress of Mathematics, Paris 1992, A. Joseph et al. (Eds), Progress in Math., Vol 119, Birkhäuser, 1994, pp. 321-370.

[5] A. Björner and H. Eriksson, Packing complexes, preprint 1999.

[6] A. Björner, L. Lovász, S.T. Vrećica and R.T. Živaljević, Chessboard complexes and matching complexes, J. London Math. Soc. 49 (1994), 25-39.

[7] A. Björner, M.L. Wachs and V. Welker, Poset fiber theorems, preprint 2002.

[8] A. Björner and M.L. Wachs, Shellable nonpure complexes and posets. I, Trans. Amer. Math. Soc. 348 (1996), 1299-1327.

[9] A. Björner and V. Welker, The homology of " $k$-equal" manifolds and related partition lattices, Adv. Math. 110 (1995), 277-313. 
[10] A. Björner and V. Welker, Complexes of directed graphs, SIAM J. Discrete Math. 12 (1999), 413-424.

[11] S. Bouc, Homologie de certains ensembles de 2-sous-groups des groupes symétriques, J. Algebra 150 (1992), 158-186.

[12] A.E. Browdy, The (co)homology of the lattices of partitions with restricted block size, Ph.D. Thesis, University of Miami, 1996.

[13] A.E. Browdy and M.L. Wachs, On the (co)homology of the lattice of partitions with restricted block size, in preparation.

[14] K.S. Brown, Euler characteristics of discrete groups and $G$-spaces, Invent. Math. 27 (1974), 229-264.

[15] K.S. Brown, Euler characteristics of groups: the p-fractional part, Invent. Math. 29 (1975), 1-5.

[16] W. Bruns and J. Herzog, Semigroup rings and simplicial complexes, J. Pure and Applied Algebra 122 (1997), 185-208.

[17] W.H. Burge, Four correspondences between graphs and generalized Young tableaux, J. Combin. Th. A 17 (1974), 12-30.

[18] A.R. Calderbank, P. Hanlon and R.W. Robinson, Partitions into even and odd block size and some unusual characters of the symmetric groups, Proc. London Math. Soc. 53 (1986), 288-320.

[19] X. Dong, Topology of bounded-degree graph complexes, to appear (preprint 2000).

[20] X. Dong, Topology of bounded-degree graph complexes, Ph.D. Thesis, University of Minnesota, 2001.

[21] X. Dong and M.L. Wachs, Combinatorial Laplacian of the matching complex, Electronic J. Combin., to appear (preprint 2000).

[22] J.-G. Dumas, F. Heckenbach, D. Saunders and V. Welker, Homology software, http://www.cis.udel.edu/ heckenba.

[23] R. Forman, Morse theory for cell complexes, Adv. Math. 134 (1998), 90-145.

[24] J. Friedman and P. Hanlon, On the Betti numbers of chessboard complexes, J. Alg. Comb. 8 (1998), 193-203.

[25] W. Fulton, Young Tableau, London Mathematical Society Student Texts 35, Cambridge University Press, 1997.

[26] P.F. Garst, Cohen-Macaulay complexes and group actions, Ph.D. Thesis, University of Wisconsin-Madison, 1979.

[27] T. Józefiak, P. Pragacz and J. Weyman, Resolutions of determinantal varieties and tensor complexes associated with symmetric and antisymmetric matrices, Astérisque 87-88 (1981), 109-189.

[28] T. Józefiak and J. Weyman, Representation-theoretic interpretation of a formula of D.E. Littlewood, Math. Proc. Cambridge Philos. Soc. 103 (1988), 193-196.

[29] J. Kahn, M. Saks and D. Sturtevant A topological approach to evasiveness, Combinatorica 4 (1984), 297-306.

[30] D.B. Karaguezian, Homology of complexes of degree one graphs, Ph.D. Thesis, Stanford University, 1994.

[31] D.B. Karaguezian, V. Reiner and M.L. Wachs, Matching complexes, bounded degree graph complexes, and weight spaces of $G L_{n}$-complexes, J. Algebra 239 (2001), 77-92.

[32] B. Kostant, Lie algebra cohomology and the generalized Borel-Weil theorem, Ann. Math. 74, (1961), 329-387. 
[33] R. Ksontini, Propriétés homotopiques du complexe de Quillen du groupe symétrique, Thèse de Doctorate, Université de Lausanne, 2000.

[34] D.E. Littlewood, The Theory of Group Characters and Matrix Representations of Groups, 2nd ed., Oxford University Press, 1950.

[35] I.G. Macdonald, Symmetric Functions and Hall Polynomials, 2nd ed., Oxford University Press, 1995.

[36] D. Quillen Homotopy properties of the poset of nontrivial p-subgroups of a group, Advances in Math. 28 (1978), 101-128.

[37] V. Reiner and J. Roberts, Minimal resolutions and homology of chessboard and matching complexes, J. Algebraic Combinatorics 11 (2000), 135-154.

[38] J. Shareshian, Some results on hypergraph matching complexes and p-group complexes of symmetric groups, preprint 2000.

[39] J. Shareshian and M.L. Wachs, Homology of matching and chessboard complexes, extended abstract, Formal Power Series and Algebraic Combinatorics, 13th International Conference, Arizona State University, Tempe, 2001.

[40] J. Shareshian and M.L. Wachs, Torsion in the homology of matching and chessboard complexes, in preparation.

[41] J. Shareshian and M.L. Wachs, Top homology of hypergraph matching complexes, p-cycle complexes and Quillen complexes of symmetric groups, in preparation.

[42] S. Sigg, Laplacian and homology of free two-step nilpotent Lie algebras, J. Algebra 185 (1996), 144-161.

[43] R.P. Stanley, Enumerative Combinatorics, Vol. 2, Cambridge University Press, 1999.

[44] S. Sundaram, Applications of the Hopf trace formula to computing homology representations, Jerusalem combinatorics '93, 277-309, Contemp. Math., 178 Amer. Math. Soc., Providence, RI, 1994.

[45] S. Sundaram, On the topology of two partition posets with forbidden block sizes, J. Pure Appl. Algebra 155 (2001), 271-304.

[46] S. Sundaram and M.L. Wachs, The homology representations of the k-equal partition lattice, Trans. Amer. Math. Soc. 349 (1997), 935-954.

[47] V.A. Vassiliev, Complexes of connected graphs, The Gelfand Mathematical Seminars, 1990-1992, 223-235, Birkhüser Boston, Boston, MA, 1993.

[48] V.A. Vassiliev, Topology of two-connected graphs and homology of spaces of knots, Differential and symplectic topology of knots and curves, 253-286, Amer. Math. Soc. Transl. Ser. 2, 190, Amer. Math. Soc., Providence, RI, 1999.

[49] M.L. Wachs, A basis for the homology of the d-divisible partition lattice, Adv. Math. 117 (1996), 294-318.

[50] M.L. Wachs, Whitney homology of semipure shellable posets, J. Algebraic Combin. 9 (1999), 173-207.

[51] M.L. Wachs, Bounded degree digraph and multigraph complexes, in preparation.

[52] V. Welker, G.M. Ziegler and R.T. Živaljević, Homotopy colimits - comparison lemmas for combinatorial applications, J. Reine Angew. Mathematik (Crelles Journal) 509 (1999), 117-149.

[53] G.M. Ziegler, Shellability of chessboard complexes, Israel J. Math. 87 (1994), 97-110. 


\section{WACHS}

[54] R.T. Živaljević and S.T. Vrećica, The colored Tverberg problem and complexes of injective functions, J. Combin. Theory A 61 (1992), 309-318.

Department of Mathematics, University of Miami, Coral Gables, FL 33124

E-mail address: wachs@math.miami.edu 